\title{
Resolución espacial en la ablación láser acoplada a la espectrometría de masas con fuente de plasma de acoplamiento inductivo ${ }^{(\cdot)}$
}

\author{
A. G.-Coedo* y M.T. Dorado*
}

\begin{abstract}
Resumen
La ablación láser como sistema de introducción de muestra a la espectrometría de masas con fuente de plasma de acoplamiento inductivo (LA-ICP-MS) ofrece la posibilidad de conocer la distribución espacial de los elementos. Variando la posición del haz láser sobre la superficie, se obtiene el perfil de la distribución lateral de elementos y, haciendo incidir sucesivos pulsos sobre un punto fijo, se obtiene el perfil de concentraciones en profundidad. Después de optimizar los parámetros operatorios se ha aplicado la técnica a muestras con distintas composiciones tanto en superficie como en profundidad. Con relación a la resolución lateral se ha comprobado que en una longitud similar al diámetro del cráter del haz láser, las señales de los elementos presentes aparecen mezcladas lo que dificulta establecer con precisión la interfase. En cuanto a la resolución en profundidad se establece claramente la influencia de la abundancia natural de los isótopos medidos.
\end{abstract}

Palabras clave Ablación láser; Espectrometría de masas con fuente de plasma de acoplamiento inductivo; Resolución espacial; Muestras soldadas; Muestras metálicas recubiertas.

\section{Spatial resolution in laser ablation inductively coupled plasma mass spectrometry}

Abstract

Keywords

\begin{abstract}
Laser ablation as sampling system in inductively coupled plasma mass spectrometry (LA-ICP-MS) offers the possibility to know the spatial distribution of elements present in a solid sample. By varying the position of the laser beam on the sample surface, the profile of the lateral distribution of elements is obtained and, with successive pulses fired on a fixed point, profile in depth is achieved. After optimization of operating parameters the technique has been applied to samples with different compositions in both surface and depth. With regard to the lateral resolution has been found that in a length similar to the crater diameter of the laser beam, the signals of the elements appear mixed, making difficult to accurately establish the interface. Regarding the resolution in depth is clearly established the influence of the natural abundance of the measured isotopes.
\end{abstract}

Laser ablation; Inductively coupled plasma mass spectrometry; Spatial resolution; Welded samples; Coated metal samples

\section{INTRODUCCIÓN}

La ablación láser (LA), como técnica de introducción de muestra en estado sólido, es objeto de numerosos estudios y desarrollos. Conceptualmente, la ablación láser es un proceso muy simple: un haz láser pulsado de alta potencia se focaliza sobre la superficie de la muestra en una atmósfera de gas inerte a presión normal; el haz láser convierte, instantáneamente, un volumen finito de la muestra sólida en un aerosol de los constituyentes. Durante el proceso de ablación la radiación láser es absorbida por los electrones en la capa superficial de la muestra, dependiendo la penetración en profundidad tanto de las propiedades físico químicas de la muestra, como de las características del láser. Los electrones de mayor temperatura abandonan la muestra de inmediato (en el orden de femtosegundos) y el resto calientan el volumen irradiado mediante colisiones con átomos de la muestra sólida (en el orden de picosegundos). Como consecuencia, se produce una fusión de la muestra en el foco de la radiación láser y, a intensidades

\footnotetext{
(•) Trabajo recibido el día 24 de marzo de 2009 y aceptado en su forma final el día 24 de noviembre de 2009.

* Centro Nacional de Investigaciones Metalúrgicas (CSIC). Gregorio del Amo 8, 28040 Madrid. Spain. coedo@cenim.csic.es.
} 
suficientemente altas, una evaporación de la muestra líquida en forma de un plasma con electrones, iones, moléculas y aglomerados ${ }^{[1]}$. El aerosol generado por el haz láser se arrastra mediante una corriente de argón a la fuente de iones, ICP, donde se vaporiza, atomiza e ioniza. Finalmente, los iones cargados positivamente se analizan en el espectrómetro de masas (LA-ICP-MS) ${ }^{[2]}$.

La técnica es especialmente interesante cuando se requiere información de la distribución espacial de la composición elemental en materiales de alta tecnología industrial. Tanto si las muestras son monocapas o multi-capas y lo mismo si están dopadas con elementos específicos o si presentan heterogeneidades, puede ser de gran utilidad obtener información sobre la distribución lateral e identificar gradientes de concentraciones en función de la profundidad. Obtener información de la distribución espacial de los componentes de determinados materiales industriales presenta un gran interés, tanto cuando las muestras presentan una diferencia de concentraciones en superficie (como es el caso de metales soldados) como en profundidad (como es el caso de materiales recubiertos). En cualquier caso, se requiere una técnica analítica con buena resolución espacial. La combinación de un haz láser focalizado con un sistema de movilidad en las tres dimensiones, con relación a la superficie de la muestra, permite realizar análisis locales de materiales heterogéneos ${ }^{[3]}$. La estrategia de muestreo regula la señal ICP-MS en función del tiempo y, de ella, depende el rendimiento analítico (fraccionamiento, precisión, exactitud). Sin duda, una de las mayores limitaciones de la técnica es el efecto de no-estequiometría en las señales transitorias, definido como fraccionamiento ${ }^{[4}$ y 5$]$.

Las estrategias de muestreo incluyen el rastreo en superficie (barrido) y el perfil en profundidad (punto fijo). En modo "barrido", con la muestra en movimiento, la posición del haz láser va variando con relación a la superficie de la misma y se obtiene el perfil de la distribución de elementos a lo largo y ancho de un área determinada. En modo "punto fijo", con el haz láser focalizado en un punto determinado de la muestra durante un cierto periodo de tiempo, se obtiene el perfil de distribución en profundidad ${ }^{[6]}$. Adquiriendo señales transitorias durante la ablación de líneas paralelas sobre la superficie de la muestra, se puede obtener una imagen de distribución elemental en un área determinada. Realizando repetitivos barridos sobre una misma línea se puede obtener una distribución tridimensional.

El proceso de ablación de sólidos es extremadamente complejo; dicho proceso depende de numerosos parámetros que están interrelacionados. En un supuesto ideal, cada pulso láser debería arrancar una cantidad reproducible de muestra y la relación entre la profundidad del cráter y el número de pulsos debería ser lineal. Sin embargo, en la práctica, la velocidad de ablación real depende del material y de diversos parámetros críticos, tales como: tipo de láser, irradiancia, relación diámetro/profundidad del cráter y fenómenos de fraccionamiento, los cuales modifican la morfología del cráter ${ }^{[7]}$. Una cuantificación de heterogeneidades en superficie, a partir de señales transitorias, es posible siempre que la resolución espacial requerida exceda 2-3 veces el diámetro del impacto láser.

En comparación con otras técnicas analíticas con buena resolución espacial, LA-ICP-MS presenta como ventajas: su rapidez, que el proceso de ablación tiene lugar a presión atmosférica, las escasas restricciones en relación con la geometría de la muestra y la posibilidad de medir simultáneamente un amplio rango de concentraciones. Como principal desventaja de la técnica hay que señalar la dificultad para evaluar las señales de ICP-MS, debido fundamentalmente a la influencia de la matriz en la ablación, a los fenómenos de fraccionamiento y a la mezcla del material durante su transporte desde la cámara de ablación hasta el sistema detector. Bleiner et al. ${ }^{[8]}$ detallan los principios y capacidades de las técnicas analíticas con resolución espacial más comunes: espectroscopia de fotoemisión (XPS), espectroscopia de electrones Auger (AES), espectrometría de masas de iones secundarios (SIMS), espectrometría de masas de neutrones secundarios (SNMS), espectrometría óptica con fuente de descarga luminiscente (GD-OES), espectrometría de masas con fuente de descarga luminiscente (GD-MS), microscopía electrónica de barrido con analizador de energías dispersivas de $\mathrm{Rx}$ (SEM-EDX) y espectrometría de masas con fuente de plasma acoplado por inducción y ablación por láser (LA-ICP-MS), comparando su resolución espacial y su capacidad de detección. Algunas de ellas (XPS, AES, SIMS, SNMS), presentan una resolución en el orden de los nanómetros, pero solo ofrecen tendencias cualitativas. LA-ICP-MS se presenta como una técnica idónea para el análisis con resolución espacial, al combinar el gran poder de detección de la espectrometría de masas con fuente de plasma, con la resolución espacial de un haz láser (10-100 $\mu \mathrm{m}$ lateral y $0.1-1 \mu \mathrm{m}$ en profundidad). Son muchas las publicaciones aparecidas en los últimos años, en relación con el análisis con resolución espacial, para una gran diversidad de materiales. Concretamente, en el campo de los materiales metálicos se han realizado diversos estudios, tanto para identificar inclusiones o heterogeneidades, como capas de recubrimiento. En relación a la resolución lateral, Dubuisson et al ${ }^{[9]}$ han evaluado el potencial de la técnica para establecer la homogeneidad de los denominados "aceros limpios", 
identificando inclusiones del tipo: $\mathrm{Mn}-\mathrm{S}, \mathrm{Mg}-\mathrm{Al}$ y Mg-Al-Ca-Ti-Si. Nafisi et al. ${ }^{[10]}$ presentan un método de barrido lineal que permite controlar las segregaciones de partículas de -Al formadas durante la solidificación de aleaciones de $\mathrm{Al}-\mathrm{Si}$, mostrando que mediante calibración externa e integrando zonas de 1-5 $\mu \mathrm{m}$, se pueden identificar segregaciones en la escala 6-7 $\mu \mathrm{m}$ con errores $<25 \%$. Karasev et al. ${ }^{[11}$ y 12] evalúan el potencial de la técnica para estudiar inclusiones en metales y otros materiales, demostrando que permite diferenciar el contenido total e insoluble de los elementos, así como la composición de inclusiones con diámetro de partícula entre 1-100 $\mu \mathrm{m}$.; en aceros, analizan cuantitativamente inclusiones de $\mathrm{Al}_{2} \mathrm{O}_{3}-\mathrm{TiN}_{\text {y }} \mathrm{Ce}_{2} \mathrm{O}_{3}-\mathrm{TiN}$ (en el rango $5-50 \mu \mathrm{m}$ ) y comparan los resultados con los obtenidos por EP$\mathrm{MA}$, mostrando que, para inclusiones complejas, los resultados mediante LA-ICP-MS son más concluyentes. Pisonero et al. ${ }^{[13]}$ han aplicado la técnica para estudiar la distribución espacial y la concentración de impurezas en silicio de grado metalúrgico; seleccionando un impacto láser de $120 \mu \mathrm{m}$ de diámetro como compromiso entre sensibilidad y resolución lateral, para muestras de tamaño de grano entre $250-500 \mu \mathrm{m}$. Latkoczy et al. ${ }^{[14]}$ han desarrollado un sistema simultáneo LIBS- LA-ICP-MS, demostrando la utilidad del sistema para caracterizar cuantitativamente la distribución lateral, en el rango micrométrico, de elementos mayoritarios y trazas en aleaciones multi-fase de magnesio; Coedo et al. ${ }^{[15} \mathrm{y}$ ${ }^{16]}$ cuantifican el aluminio y titanio, soluble y total, en aceros y preparan muestras sintéticas para identificar el tamaño de partícula de las inclusiones de $\mathrm{Al}_{2} \mathrm{O}_{3}$ en aceros, encontrando una correlación lineal entre área de pico y tamaño medio de partícula (en el intervalo:25-100 $\mathrm{m}$ ). Con relación a perfiles en profundidad, Plotnikov et al..$^{[17]}$ y Bleiner et al. ${ }^{[18]}$ han realizado estudios sobre substratos de acero y WC/Co recubiertos con capas de $\mathrm{TiC}, \mathrm{TiN}, \mathrm{Ti}(\mathrm{C}, \mathrm{N})$ y $(\mathrm{Ti}, \mathrm{Al}) \mathrm{N}$; utilizando un espectrómetro de masas quadrupolo y uno de tiempo de vuelo, respectivamente. Con el quadrupolo obtienen una resolución en profundidad $\leq 25 \mu \mathrm{m}$ (calculada a partir de las curvas intensidadtiempo), mientras que con el de tiempo de vuelo llegan a una resolución de 0,20 $\mu \mathrm{m}$ por pulso láser. Kanicky et al. ${ }^{[19]}$, utilizando un láser excimer de $\mathrm{ArF}^{*}$ (193 nm), han demostrado la dependencia de la resolución en profundidad con la relación diámetro/profundidad del cráter, para aceros recubiertos con capas de $\mathrm{ZrN}$ y TiN, obteniendo un valor óptimo de resolución en profundidad de $0,6 \mu \mathrm{m}$, para cráteres de $20 \mu \mathrm{m}$ de diámetro. Coedo et al. ${ }^{[20]}$ han evaluado la resolución en profundidad, en una serie de muestras de aceros recubiertas con capas de cobre, entre 5 y $200 \mu \mathrm{m}$, alcanzando valores de resolución en profundidad entre 7 y 9 veces por debajo del correspondiente valor del espesor de capa. Izmir et al. ${ }^{[21]}$ comparan el uso de los sistemas de "barrido" y "punto fijo" para determinar la difusión en la interfase de recubrimientos de NiCrAlY sobre aleaciones de alta temperatura, observando tanto la existencia de una difusión del aluminio de la capa hacia el substrato, como la difusión de algunos de los aleantes del substrato (cobalto, tántalo, molibdeno, wolframio...) hacia el recubrimiento. Hrdlicka et al. ${ }^{[22]}$ estudian la capacidad de la técnica para el análisis de recubrimientos de zinc; utilizan dos tipos de láser: $\mathrm{Nd}$ :YAG (1064 nm) y ArF* (193 nm), obteniendo valores de velocidad de ablación de $0,10 \mu \mathrm{m}$ por pulso para el láser UV y de 0,06 $\mu \mathrm{m}$ por pulso para el IR, alcanzando una resolución en profundidad de varios $\mu \mathrm{m}$. Lieve et al. ${ }^{[23]}$ estudian en profundidad los perfiles de materiales industriales de alta tecnología, con capas de recubrimiento entre 1 y $200 \mu \mathrm{m}$, utilizando un láser de $\operatorname{ArF}^{*}(193 \mathrm{~nm})$. Mateo et al. ${ }^{[24]}$ analizan aceros recubiertos con polímeros, utilizan un láser con anchura de pulso de 120 femtosegundos, y obtienen una resolución de $240 \mathrm{~nm}$ para un acero galvanizado y de 2,3 $\mu \mathrm{m}$ para un recubrimiento polímero-polímero sobre galvanizado. Sarah et al. ${ }^{[25]}$ han aplicado la técnica al estudio de monedas antiguas, desarrollado un protocolo que permite la realización de perfiles de concentración en profundidad, tanto para los elementos mayoritarios (plata y cobre) como para los minoritarios (zinc, oro, plomo, bismuto), verificado el enriquecimiento superficial en plata.

En este estudio se evalúa la técnica LA-ICP-MS con respecto a su resolución lateral, mediante el estudio de diversos tipos de muestras de metales soldados y a su resolución en profundidad, a partir de distintas muestras con capas y substratos metálicos.

\section{EXPERIMENTAL}

\subsection{Equipos}

Se ha utilizado un láser tipo Nd:YAG en modo Qswitch, con una distribución gausiana de energía, operando a 266 nm (LSX-100, CETAC Technologies, Omaha, Nebraska), acoplado a un Espectrómetro de Masas Quadrupolo con fuente de Plasma de Acoplamiento Inductivo (ELAN 6000, Perkin Elmer, SCIEX, Ontario, Canadá).

El porta muestras del módulo láser está provisto de un control computerizado de la posición de la muestra con respecto al haz láser, mediante etapas de traslación, $x-y-z$; la etapa de traslación, $x-y$, controla la posición del haz en la superficie de la 
muestra y el eje, $z$, permite posicionar el enfoque del haz sobre la misma. El sistema dispone de una cámara-microscopio que permite visualizar las áreas de interés en la muestra, así como posicionar el punto de impacto láser y su focalización.

La energía del pulso láser se ha medido con un medidor Molectron Detector, Inc. (USA), modelo EM 400. La morfología de los cráteres se ha observado mediante microscopía electrónica de barrido, SEM (DSM 400, Zeiss, Germany) y la morfología de los surcos de barrido se ha evaluado con microscopio confocal e interferométrico (SENSOFAR, PL $\mu$ 2300).

Los parámetros operatorios tanto del sistema láser como del ICP-MS fueron optimizados tanto en cuanto a sensibilidad como a estabilidad. Todos los datos han sido recogidos a partir de señales transitorias, en modo altura de pico y con un punto por pico. Las señales fueron adquiridas y visualizadas en función del tiempo y su monitorización en tiempo real ha permitido modificar los parámetros durante el análisis y así establecer los valores óptimos. La tabla I recoge las condiciones operatorias de los equipos de Ablación por Láser y del Espectrómetro ICP-MS.

\subsection{Muestras}

Para el estudio de la resolución lateral se han preparado muestras de aceros de distintos tipos, soldados mediante soldaduras con arco y con láser, y una muestra de zinc con cobre insertado; y, para el estudio de la resolución en profundidad, se han preparado muestras con diversos substratos y recubrimientos metálicos.

\subsubsection{Muestras de aceros soldados con arco}

Se ha seguido el procedimiento de soldadura con arco (90-100 A, $24 \mathrm{~V})$, con diseño convencional, en $\mathrm{V}$ para los aceros al carbono y en $\mathrm{X}$ para $1,1 \% \mathrm{Cr}$ y acero al carbono $(0,1 \% \mathrm{Si} ; 0,67 \% \mathrm{Mn} ; 0,01 \% \mathrm{Cr})$, cordón de soldadura: acero al carbono $(0,6 \% \mathrm{Si}$; $1,2 \% \mathrm{Mn} ; 0,06 \% \mathrm{Cr}$ ); acero Inoxidable AISI 316 (18\% Cr; $10 \% \mathrm{Ni} ; 3 \% \mathrm{Mo}$ ? $67 \% \mathrm{Fe}$ ) y acero al carbono (? $90 \% \mathrm{Fe}$; 0,15\% Cr), cordón de soldadura: acero INOX (16\% Cr; 8 \% Ni; 2,5\% Mo; ? $71 \%$ $\mathrm{Fe})$.

\subsubsection{Aceros INOX soldados con láser}

Se ha empleado un láser pulsado Nd-YAG (RofinSinar RSY-1000P), con guía de fibra óptica, con 36 J de energía por pulso, 9,6 ms de anchura de pulso, $26,7 \mathrm{~Hz}$ de frecuencia y a una velocidad de soldadura: $0,45 \mathrm{~m} / \mathrm{min}$. Se han preparado 5 muestras, soldando un acero austenítico (A) y un acero ferrítico (F). El haz láser se posicionó a diferentes distancias de la junta de unión de los dos aceros: focalización del haz láser justo en la unión (muestra 0); focalización a 0,2 y 0,4 mm de la junta de unión, en la zona del acero austenítico (muestras: $+0,2$ y $+0,4$ ); focalización a 0,2 y $0, .4 \mathrm{~mm}$ de la junta de unión, en la zona

Tabla I. Condiciones operatorias de LA-ICP-MS

Table I . LA-ICP-MS operating conditions

ICP-MS (ELAN 6000) $\quad$ LA (LSX 100)

\author{
Potencia: 1200W \\ Caudal de Ar: 14 l/min \\ Caudal de Ar de transporte: $0.65 \mathrm{l} / \mathrm{min}$ \\ Frecuencia ICP: $40.86 \mathrm{MHz}$ \\ Detector de Masas: modo Dual \\ Tiempo de lectura por analito: $10 \mathrm{~ms}$ \\ Barridos/Lectura: 4 \\ Lecturas/Réplica: el $n^{\circ}$ de lecturas por \\ réplica debe ajustarse en cada caso, de \\ modo que el tiempo de réplica sea suficiente \\ para aplicar el número de pulsos láser \\ requeridos.
}

Tipo de Láser: pulsado de Nd-YAG

Modo de Láser: $Q$ switched

Distribución de la energía: Gausiana

Longitud de onda: $266 \mathrm{~nm}$ (UV)

Anchura de pulso: 8 ns

Energía de salida: entre 0.4 y $4.0 \mathrm{~mJ} /$ pulso

Frecuencia de repetición: $1-20 \mathrm{~Hz}$

Método: Punto fijo y barrido

Transporte desde la cámara LA al ICP-MS:

Caudal de Ar de transporte: $0.65 \mathrm{l} / \mathrm{min}$;

Conducción: tubo tygon $(40 \mathrm{~cm} / 5 \mathrm{~mm}$ id). 
del acero ferrítico (muestras: $-0,2$ y 0,4 ). El ancho resultante de todos los cordones de soldadura fue de 2,2 $\pm 0,2 \mathrm{~mm}$. La composición de ambas muestras de acero se recoge en la tabla II.

\subsubsection{Muestra de cobre insertado en zinc}

Se ha preparado una muestra, insertando mecánicamente un cilindro de cobre, de $4 \mathrm{~mm}$ de diámetro, en una muestra de zinc, para comprobar el comportamiento de las medidas cuando se tiene la seguridad de que no existe difusión de elementos de un material al otro.

\subsubsection{Muestras con substratos y recubrimientos metálicos}

Se han preparado muestras con diferentes substratos y recubrimientos: Substratos de cobre, aluminio y cromo recubiertos con una capa de níquel $(25,30$ y $20 \mu \mathrm{m}$ de espesor, respectivamente) y substratos de acero con recubrimientos de cobre y zinc ( 22 y 25 $\mu \mathrm{m}$, respectivamente). Dichos espesores han sido verificados por espectroscopia de emisión con fuente de descarga luminiscente (GD-OES).

\section{RESULTADOS Y DISCUSIÓN}

\subsection{Estudio de la influencia de los parámetros sistema LA operatorios del}

En la ablación láser la resolución espacial, tanto lateral como en profundidad, está teóricamente condicionada por la velocidad de ablación y la relación entre la profundidad y la anchura del cráter o del surco. Si se mantiene constante la focalización y frecuencia $(\mathrm{Hz})$ del haz láser, el parámetro que condiciona todo el proceso es la energía del pulso láser. Por otra parte, la relación entre el diámetro y la profundidad del cráter, cuando se hace incidir el haz láser en un punto fijo de la superficie (SP), depende del número de pulsos; y, la relación entre la anchura y profundidad del surco, cuando se hace incidir el haz láser sobre la muestra desplazándose linealmente (scanning), depende de la velocidad de barrido. Para estudiar la influencia de estos parámetros se ha utilizado una muestra de acero al carbono, evaluando por ICP-MS las señales correspondientes al ${ }^{57} \mathrm{Fe}$, después de la sistemática optimización del equipo. Se ha estudiado el efecto de la energía del pulso láser, del número de pulsos y su focalización, tanto en el diámetro y profundidad del cráter como en la señal ICP-MS.

Se han realizado ensayos con los siguientes valores de energía: 0,6, 1,5, 2,5, 3,3 y $4 \mathrm{~mJ} /$ pulso, correspondiendo a los siguientes valores de irradiancia: 10 , $15,17,19$ y $21 \mathrm{GW} \mathrm{cm}^{-2}$. Su influencia en el diámetro del cráter se muestra en la figura 1. La influencia de las distintas energías ensayadas, en función del número de pulsos, en las señales ICP-MS se presenta en la figura 2. Como puede observarse, tanto el

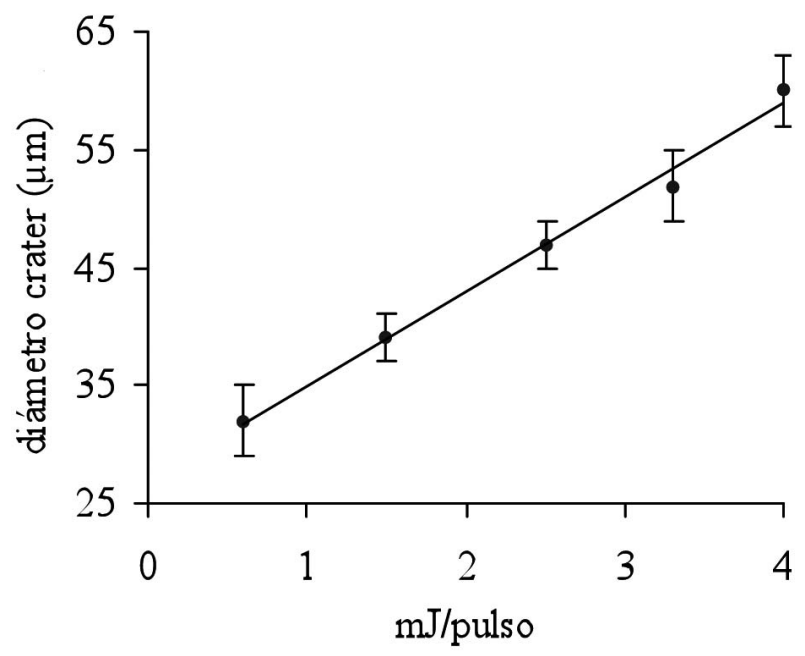

Figura 1. Influencia de la energía del pulso láser en el diámetro del cráter.

Figure 1. Influence of laser pulse energy on crater diameter size.

Tabla II. Composición de los dos aceros inoxidables empleados en las soldaduras láser (\%)

Table II. Chemical composition of stainless steels for welding laser samples (\%)

\begin{tabular}{ccccccccccccc}
\hline Muestra & $\mathbf{C}$ & $\mathbf{S i}$ & $\mathbf{M n}$ & $\mathbf{C r}$ & $\mathbf{N i}$ & $\mathbf{M o}$ & $\mathbf{P}$ & $\mathbf{S n}$ & $\mathbf{C u}$ & $\mathbf{T i}$ & $\mathbf{A l}$ & $\mathbf{A s}$ \\
\hline A & 0,020 & 0,446 & 1,42 & 18,37 & 8,98 & 0,30 & 0,028 & 0,014 & 0,30 & 0,006 & 0,009 & 0,004 \\
F & 0,047 & 0,335 & 0,43 & 16,28 & 0,13 & 0,014 & 0,019 & 0,010 & 0,053 & 0,009 & 0,000 & 0,000
\end{tabular}




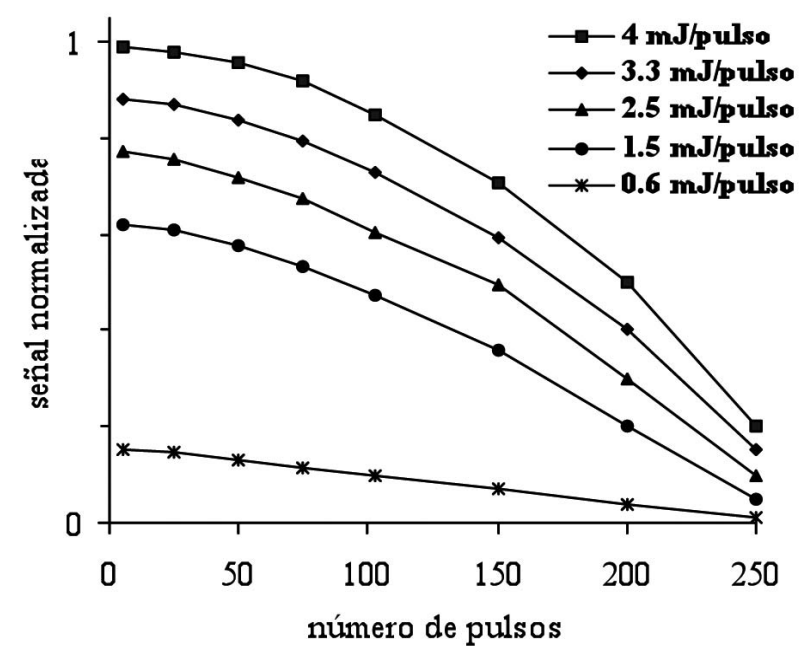

Figura 2. Efecto de la energía de pulso y del número de pulsos en las señales ICP-MS.

Figure 2. Influence of laser pulse energy and number of laser pulses on the ICP-MS signals.

diámetro del cráter como la señal ICP-MS crecen al aumentar la energía del pulso láser. Por otra parte, a partir de los 50 pulsos la señal comienza a decrecer: entre 50 y 150 pulsos la señal decrece hasta aproximadamente el $75 \%$ de su valor inicial; entre 150 y 200 pulsos prosigue el gradual descenso y a partir de los 200 pulsos la señal decrece drásticamente. La energía seleccionada para los siguientes ensayos ha sido 1,5 $\mathrm{mJ} /$ pulso, equivalente a una irradiancia de $15 \mathrm{GW}$ $\mathrm{cm}^{-2}$, dado que origina el cráter de pequeño diámetro, dando lugar a una señal con buena sensibilidad y estabilidad.

La variación del diámetro y la profundidad de penetración, en función del número de pulsos, se muestran en la figura 3 y la señal ICP-MS, en función de la relación entre la profundidad y el diámetro del cráter, se presenta en la figura 4. Como puede observarse la profundidad del cráter aumenta linealmente al aumentar el número de pulsos, mientras que el diámetro no varía. Así mismo, la señal ICP-MS decrece a medida que aumenta la relación profundidad/diámetro, siendo esta caída mucho más pronunciada a partir de una relación $\approx 2$ (la profundidad doble del diámetro). El número de pulsos que proporciona una relación $\approx 1$ (profundidad y diámetros similares) es 35 , siendo este el valor seleccionado para los ensayos siguientes.

El efecto de la focalización del haz láser con relación a la superficie de la muestra en el diámetro del cráter y consiguientemente en la densidad de energía (Irradiancia) se recoge en la figura 5. La focalización (WD) se ha estudiado entre $-5.000 \mathrm{y}+5.000 \mu \mathrm{m}$.

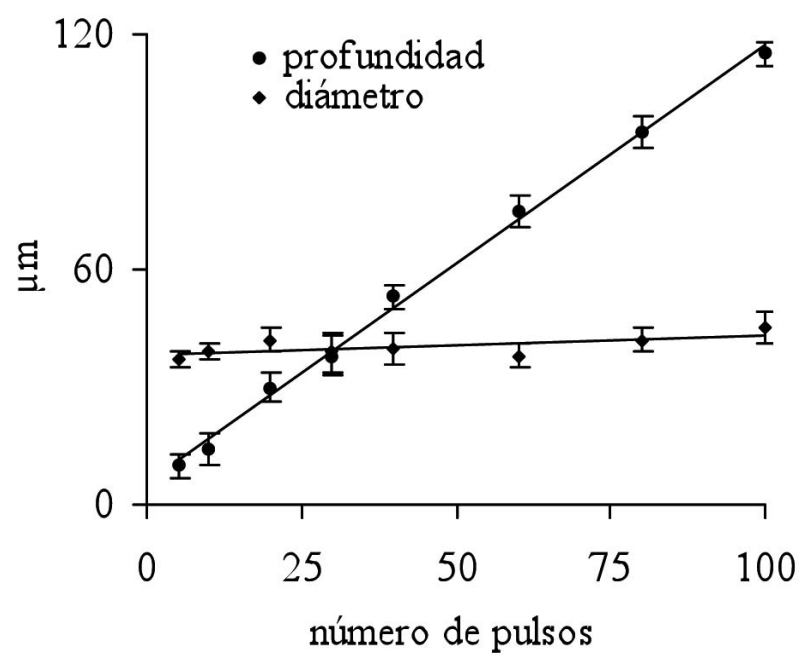

Figura 3. Variación de la profundidad y diámetro del cráter en función del número de pulsos.

Figure 3. Influence of number of laser pulses on depth and width of the crater.

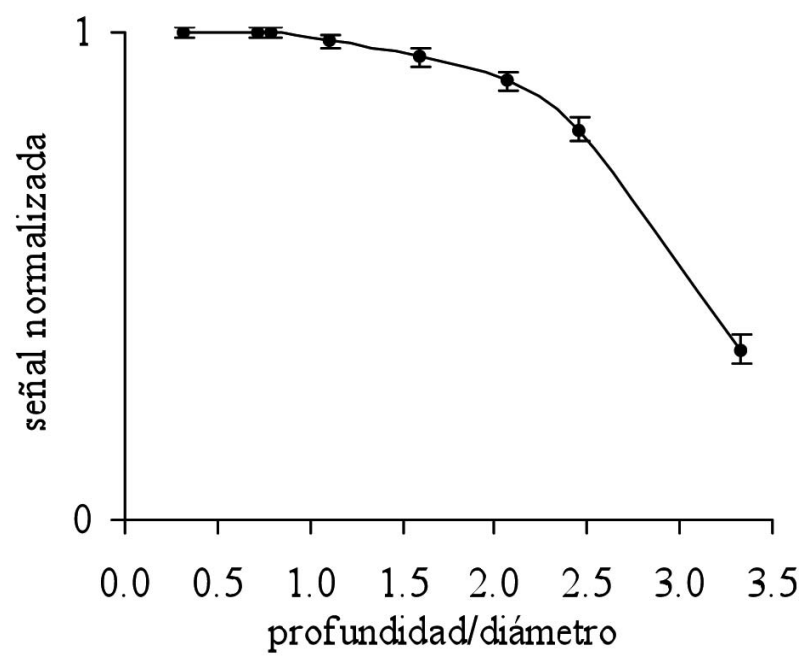

Figura 4. Variación de la señal ICP-MS en función de la relación profundidad/diámetro.

Figure 4. Influence of depth/diameter crater ratio on normalized ICP-MS signal.

Los valores positivos indican focalizaciones por encima de la superficie de la muestra y los negativos, por debajo de la misma. Como puede observarse el cráter con menor superficie y, consecuentemente, la mayor irradiancia, se consigue focalizando en la superficie de la muestra (distancia focal $=0$ ). Con cualquier otra focalización, tanto en valores positivos como negativos, la superficie de los cráteres es mayor y la densidad de energía menor. 


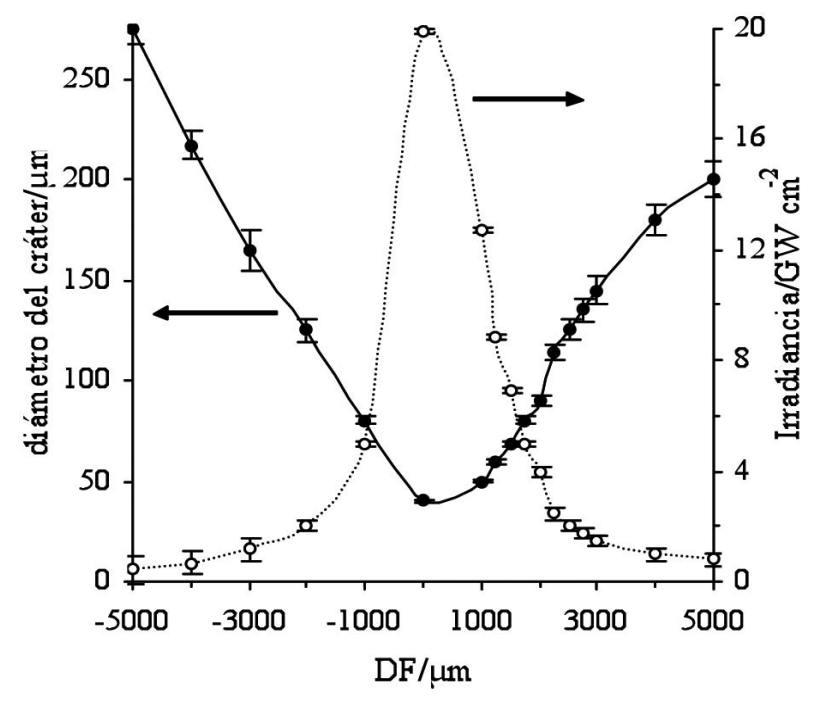

Figura 5. Influencia de la focalización en el diámetro del cráter e irradiancia.

Figure 5. Influence of focalization on crater diameter and irradiance.

\subsection{Estudios relativos a la resolución lateral}

\subsubsection{Influencia de distancia entre impactos láser y de velocidad de barrido}

Para optimizar la resolución lateral se ha estudiado la influencia de la velocidad de barrido en las señales
ICP-MS. Para evaluar éste parámetro se ha realizado un estudio previo relativo a la influencia de la distancia entre impactos láser realizados en puntos fijos. Esta influencia, realizando 4 impactos consecutivos de 35 pulsos con una energía de $1,5 \mathrm{~mJ} /$ pulso (diámetro de los cráteres $\approx 40 \mu \mathrm{m}$ ) a 40 y $20 \mu \mathrm{m}$ de distancia entre impactos, se presenta en la figura 6.

Como muestra la figura, con una distancia entre impactos, aproximadamente, igual al tamaño de los cráteres, para los distintos impactos se han obtenido señales similares. Este mismo comportamiento se observa cuando los impactos están más separados, debido a que en cada impacto incide sobre una superficie limpia. Sin embargo, cuando los impactos están separados por una distancia inferior al tamaño del cráter, la señal correspondiente al primer impacto es mayor que las correspondientes a los impactos sucesivos; esto, se debe al hecho de que en el primer impacto el número total de pulsos láser que intervienen en el cráter es de 35, mientras que en los siguientes, al estar solapados en un $50 \%$ de su superficie, es $>50(\approx 70)$, con lo que la profundidad de los cráteres consecutivos es mayor, aumentando la relación profundidad/diámetro y, consecuentemente, disminuyendo la señal ICP-MS.

La figura 7 muestra la influencia de la velocidad de barrido en la profundidad del surco y la figura 8 recoge su efecto sobre las señales ICP-MS. Como puede observarse, la profundidad del surco varía inversamente a la velocidad de barrido. Utilizando una energía láser de 1,5 mJ/pulso (ancho del surco $\approx 40 \mu \mathrm{m}$ ), se precisa aplicar una velocidad de barrido de $6 \mu \mathrm{m} \mathrm{s}^{-1}$ para conseguir un surco con una relación profundi$\mathrm{dad} /$ ancho $\approx 1$ (profundidad y ancho $\approx 40 \mu \mathrm{m}$ ). distancia entre impactos: 40 um

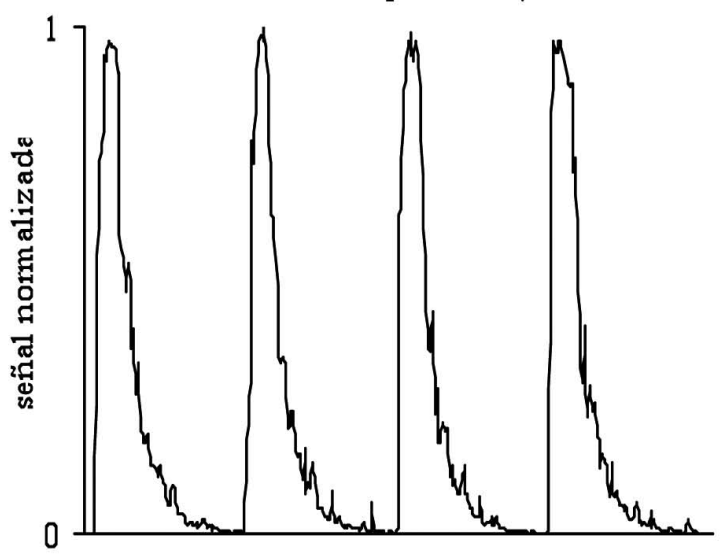

distancia entre impactos::20 $\mu \mathrm{m}$

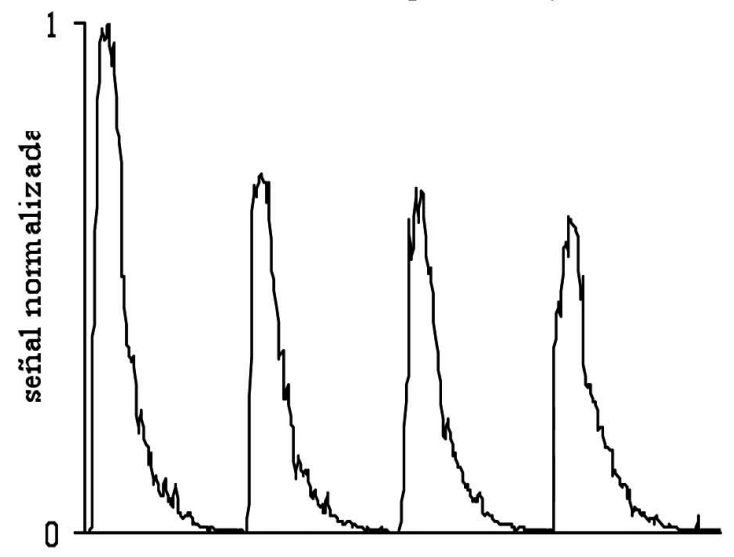

Figura 6. Señales normalizadas correspondientes a 35 pulsos láser lanzados en 4 puntos fijos, espaciados 40 y $20 \mu \mathrm{m}$, respectivamente.

Figure 6. Normalized signals corresponding to four consecutive spots (35 shots) at 40 y $20 \mu \mathrm{m}$ respectively. 


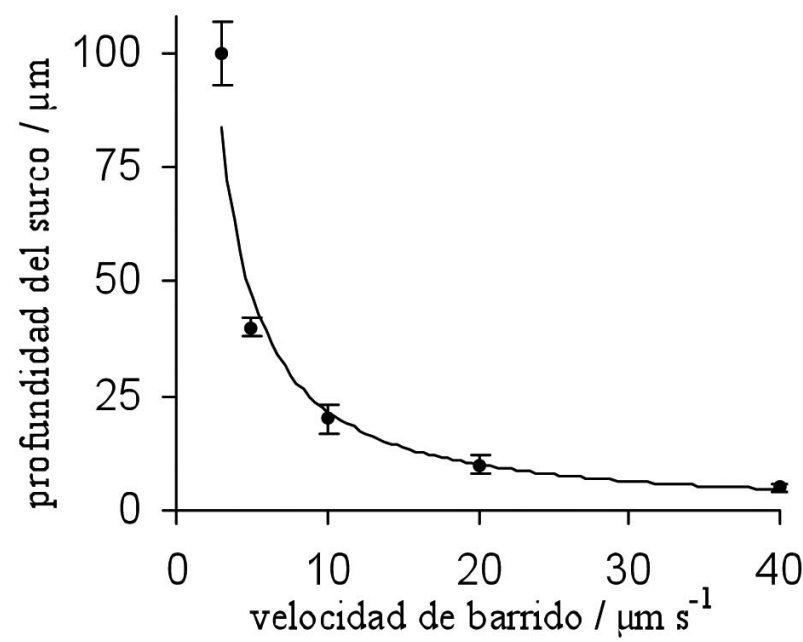

Figura 7. Profundidad del surco en función de la velocidad de barrido

Figure 7. Influence of scanning speed on depth of laser track.

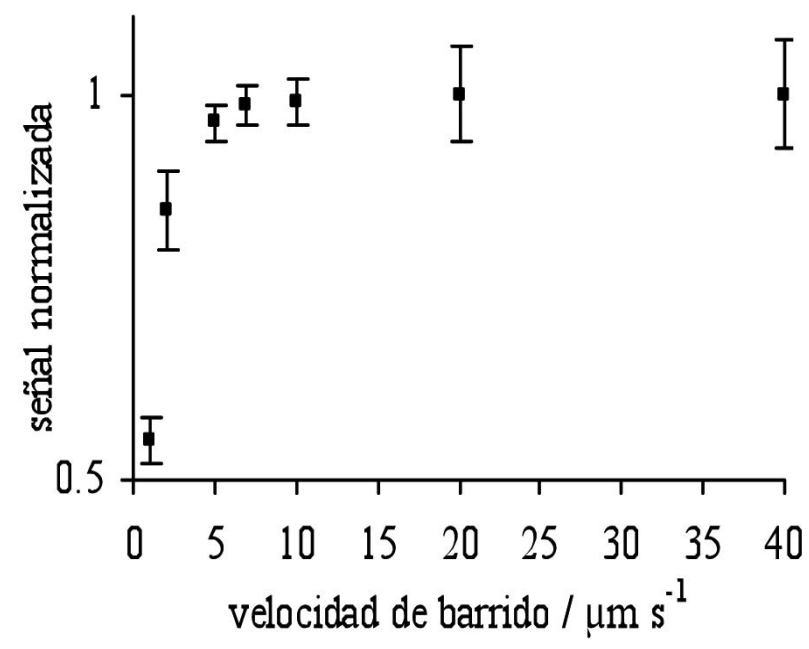

Figura 8. Intensidad de la señal ICP-MS en función de la velocidad de barrido

\section{Figure 8. Normalized ICP-MS signal vs. scanning} speed.

Operando con una frecuencia de $5 \mathrm{~Hz}$ ( 5 pulsos por segundo) la velocidad de barrido corresponde a $1 \mu \mathrm{m}$ por pulso y, consecuentemente, el surco alcanzará una profundidad constante $(\approx 40 \mu \mathrm{m})$ a los $7 \mathrm{~s}$, correspondiendo al impacto de 35 pulsos. En cuanto a la intensidad de la señal ICP-MS los valores máximos de sensibilidad se obtienen para velocidades de barrido por encima de los $5 \mu \mathrm{m} \mathrm{s}^{-1}$, cuando el solapamiento de impactos hace que la profundidad constante del surco se obtenga con un número de pulsos inferior a 40 , presentando las velocidades entre 5 y $10 \mu \mathrm{m} \mathrm{s}^{-1}$ los valores con menor dispersión $(\mathrm{RSD}<7 \%)$.

Interrelacionando el diámetro del cráter con la velocidad de barrido se observa que cuando el diámetro del cráter es mayor que la velocidad de barrido existe un solapamiento de los cráteres durante la ablación, dando lugar a un mayor número de pulsos por cráter y, por tanto, se ablaciona menos material por unidad de tiempo, disminuyendo la señal. La tabla III presenta los valores correspondientes a la proporción de cráter por pulso y al número de pulsos por cráter, en función de la velocidad de barrido y del diámetro del cráter. Los mejores resultados se obtienen combinando ambos parámetros de manera que el diámetro del cráter sea pequeño al tiempo que la señal presente una buena sensibilidad y estabilidad.

A partir de estos estudios, los valores seleccionados para los ensayos relacionados con la evaluación de la resolución lateral han sido: Energía: 1,5 mJ/pulso y velocidad: $6 \mu \mathrm{m} / \mathrm{s}$. De esta forma se consigue una proporción pequeña de cráter por segundo $(0,15)$ al tiempo que un número suficiente de pulsos por cráter para obtener una buena sensibilidad y estabilidad.

Con estas condiciones y con $\mathrm{F}=5 \mathrm{~Hz}$, cada $7 \mathrm{~s}$ se ablaciona una superficie de muestra $\approx$ al diámetro del cráter $(40 \pm 2 \mu \mathrm{m})$, siendo este el tiempo equivalente a 35 pulsos.

\subsubsection{Aplicación a muestras metálicas soldadas e insertadas}

\subsubsection{Muestras soldadas con arco}

Aplicando los parámetros operatorios optimizados, se han evaluado las señales transitorias producidas en las interfases de las muestras soldadas con diferentes tipos de aceros. La figura 9 recoge las variación de las señales de manganeso, cromo y silicio, cuando el haz láser pasa de un acero de baja aleación $(0,3 \% \mathrm{Si} ; 1,2 \% \mathrm{Mn} ; 1,1 \% \mathrm{Cr})$ al cordón de soldadura $(0,6 \% \mathrm{Si} ; 1,2 \% \mathrm{Mn} ; 0,06 \% \mathrm{Cr})$ y, a continuación, del cordón de soldadura a otro acero al carbono $(0,1 \% \mathrm{Si} ; 0,67 \% \mathrm{Mn} ; 0,01 \% \mathrm{Cr})$. El barrido se inicia $\approx 1.000 \mu \mathrm{m}$ antes del cordón $y$, una vez atravesado todo el cordón, se continúa en la zona del otro acero $\approx 1.000 \mu \mathrm{m}$.

La figura 10 recoge las variación de las señales del hierro y del cromo cuando el haz láser pasa de un acero al carbono $(\approx 90 \% \mathrm{Fe} ; 0,15 \% \mathrm{Cr})$ al cordón de soldadura INOX $(16 \% \mathrm{Cr} ; 8 \% \mathrm{Ni} ; 2,5 \% \mathrm{Mo}$; $\approx 71 \% \mathrm{Fe}$ ) y, a continuación, del cordón INOX a un acero AISI $316(18 \% \mathrm{Cr} ; 10 \% \mathrm{Ni} ; 3 \% \mathrm{Mo} ; \approx 67$ 
Tabla III. Influencia de la velocidad de barrido y del diámetro del cráter en la proporción de cráteres por segundo y en el número de pulsos por cráter

Table III. Influence of scanning speed and crater diameter on the numbers of craters per second and number of pulses per crater

\begin{tabular}{|c|c|c|c|c|c|c|}
\hline & \multirow{2}{*}{$\begin{array}{c}\text { Vel } \\
(\mu \mathrm{m} / \mathrm{s})\end{array}$} & \multicolumn{5}{|c|}{ Diámetro crater $(\mu \mathrm{m})$} \\
\hline & & 35 & 40 & 45 & 56 & 70 \\
\hline \multirow{6}{*}{$\begin{array}{l}\text { Proporción de cráter por segundo = } \\
\text { (vel. barrido/ diámetro crater) }\end{array}$} & 3 & 0,09 & 0,08 & 0,07 & 0,05 & 0,04 \\
\hline & 6 & 0,17 & 0,15 & 0,13 & 0,11 & 0,09 \\
\hline & 10 & 0,29 & 0,25 & 0,22 & 0,18 & 0,14 \\
\hline & 30 & 0,86 & 0,75 & 0,67 & 0,54 & 0,43 \\
\hline & 50 & 1,43 & 1,25 & 1,11 & 0,89 & 0,71 \\
\hline & 100 & 2,86 & 2,50 & 2,22 & 1,79 & 1,43 \\
\hline \multirow{6}{*}{$\begin{array}{l}N^{\circ} \text { de pulsos por cráter (frecuencia } 5 \\
\mathrm{~Hz})=(\text { diámetro } \times 5 / \text { vel. barrido) }\end{array}$} & 3 & 58,3 & 66,7 & 75,0 & 93,3 & 116 \\
\hline & 6 & 29,2 & 33,3 & 37,5 & 46,7 & 58,3 \\
\hline & 10 & 17,5 & 20,0 & 22,5 & 28,0 & 35,0 \\
\hline & 30 & 5,8 & 6,7 & 7,5 & 9,3 & 11,7 \\
\hline & 50 & 3,5 & 4,0 & 4,5 & 5,6 & 7,0 \\
\hline & 100 & 1,7 & 2,0 & 2,2 & 2,8 & 3,5 \\
\hline
\end{tabular}

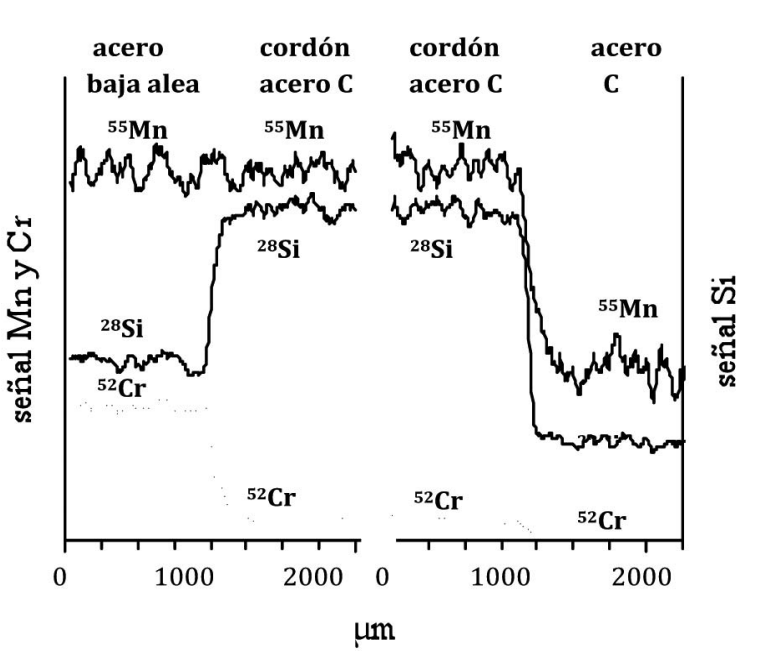

Figura 9. Señales de los isótopos ${ }^{55} \mathrm{Mn},{ }^{28} \mathrm{Si} \mathrm{y}$ ${ }^{52} \mathrm{Cr}$, cuando se realiza un barrido pasando del acero de baja aleación (1.2\% Mn, $0.3 \% \mathrm{Si}, 1.1 \%$ $\mathrm{Cr})$, atravesando el cordón $(1.2 \% \mathrm{Mn}, 0.6 \% \mathrm{Si}$, $0.06 \% \mathrm{Cr})$ al acero al C $(0.67 \% \mathrm{Mn}, 0.1 \% \mathrm{Si}$, $0.01 \% \mathrm{Cr}$ ).

Figure 9. Signals of ${ }^{55} \mathrm{Mn},{ }^{28} \mathrm{Si}$ and ${ }^{52} \mathrm{Cr}$ isotopes, during a continuous line scan ablation over weld: Low alloy steel (1.2\% Mn, 0.3\% Si, 1.1\% Cr)welded bead (1.2\% Mn, $0.6 \% \mathrm{Si}, 0.06 \% \mathrm{Cr}$ ) - C steel $(0.67 \% \mathrm{Mn}, 0.1 \% \mathrm{Si}, 0.01 \% \mathrm{Cr})$.

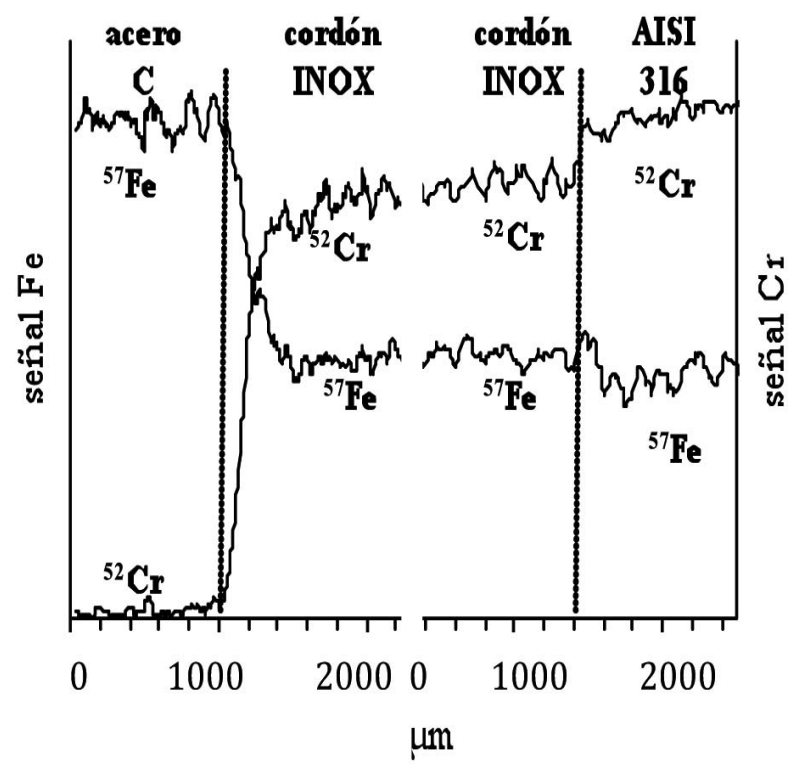

Figura 10. Señales de los isótopos ${ }^{52} \mathrm{Cr}$ y ${ }^{57} \mathrm{Fe}$, cuando se realiza un barrido pasando del acero al C ( $\approx 90 \% \mathrm{Fe}, 0.15 \% \mathrm{Cr}$ ) al acero AISI ( $\approx 67 \%$ $\mathrm{Fe}, 18 \% \mathrm{Cr}$ ), atravesando el cordón de soldadura INOX ( $\approx 71 \% \mathrm{Fe}, 16 \% \mathrm{Cr})$.

Figure 10. Signals of ${ }^{52} \mathrm{Cr}$ and ${ }^{57} \mathrm{Fe}$ isotopes, during line scan ablation over weld: $C$ steel $(\approx 90 \% \mathrm{Fe}$, $0.15 \% \mathrm{Cr}$ ) - Welded bead INOX ( $\approx 71 \% \mathrm{Fe}, 16 \%$ Cr) - Inox $A I S I$ ( $\approx 67 \% \mathrm{Fe}, 18 \% \mathrm{Cr})$. 
$\% \mathrm{Fe})$. El barrido se inicia $\approx 1.000 \mu \mathrm{m}$ antes del cordón, en la zona del acero al carbono y, una vez atravesado todo el cordón, se continúa en la zona del acero al AISI $316 \approx 1.000 \mu \mathrm{m}$. Cada isótopo se ha medido cada $156 \mathrm{~ms}$ (6 medidas por segundo) y con la velocidad de rastreo de $6 \mu \mathrm{m} / \mathrm{s}$, cada medida corresponde a $1 \mu \mathrm{m}$ de superficie de la muestra. Las figuras muestran, claramente, las diferencias de concentraciones cuando se pasa de un material a otro. En las interfases, puede apreciarse que existe una zona, de $\approx 200$ $\mu \mathrm{m}$ en el caso de un acero de baja aleación y un acero al carbono, y de $\approx 300 \mu \mathrm{m}$ en el caso de acero al carbono y acero INOX, donde se observa un gradiente de concentraciones para los elementos con distinta concentración en las muestras soldadas, debido tanto a difusión entre los metales como a fenómenos de fragmentación y transporte.

\subsubsection{Muestras soldadas con láser}

En las muestras de aceros inoxidables soldadas con láser, se ha evaluado la influencia de la posición del haz láser con respecto a la junta de unión de ambas muestras, en la composición del cordón de soldadura generado. Para ello, se ha estudiado la evolución de la señal del níquel, por ser este elemento el que presenta mayor diferencia de concentración en ambas muestras ( $8 \%$ en el acero austenítico y $0,1 \%$ en el ferrítico) La figura 11 muestra la respuesta de la señal del isótopo ${ }^{60} \mathrm{Ni}$ en las muestras soldadas con el haz posicionado en la unión (muestra: 0), con el haz posicionado a $0,4 \mathrm{~mm}$ de la unión, en la zona del acero austenítico (muestra: $+0,4$.) y con el haz posicionado a $0,4 \mathrm{~mm}$ en la zona del acero ferrítico (muestra: $-0,4)$ ). Las medidas se han realizado empezando a lanzar los impactos láser, aproximadamente a $600 \mu \mathrm{m}$ antes del cordón de soldadura, en la muestra austenítica, atravesándolo completamente $(\approx 2,0 \mathrm{~mm})$ y continuando durante otros $600 \mu \mathrm{m}$ en la muestra ferrítica. Como puede observarse, cuando el haz láser se posiciona justo en la unión, la concentración de níquel en el cordón es la media de las concentraciones en ambas muestras. Dependiendo de su posicionamiento sobre una u otra muestra, la composición del cordón es proporcional a la mayor o menor aportación de cada una de ellas. Como en el caso de los aceros soldados con arco, en las dos interfases del paso del acero austenítico al cordón y del cordón al acero ferrítico, existe una zona donde se observa un gradiente de concentraciones. En dicha zona, las señales obtenidas no permiten establecer con seguridad que parte se debe a difusión entre metales o a fenómenos de fragmentación y transporte.
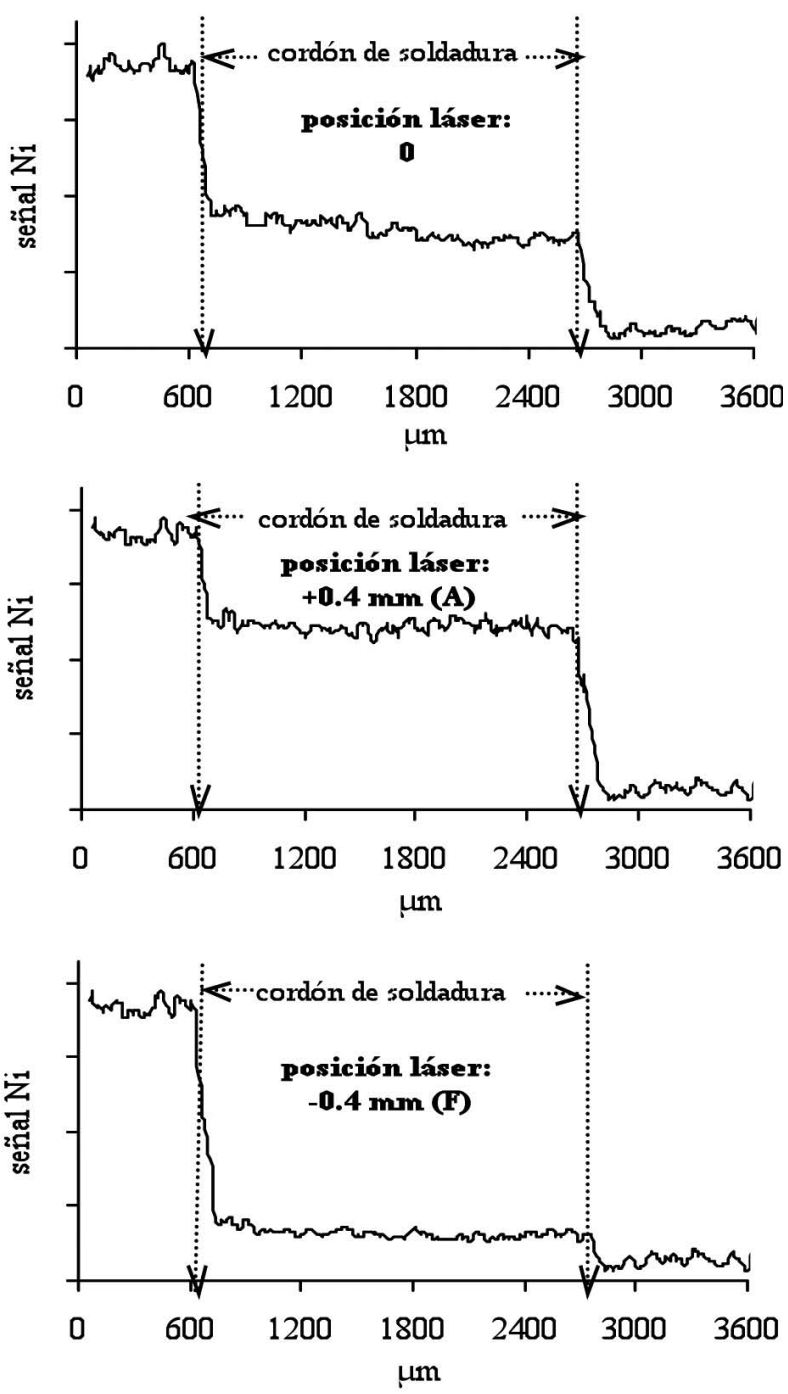

Figura 11. Variación de las señales del ${ }^{60} \mathrm{Ni}$ en función de la posición del haz láser en la soldadura.

Figure 11. ${ }^{60} \mathrm{Ni}$ signals variation vs. position of the laser beam over welded bead.

\subsubsection{Muestra insertada}

Para comprobar el comportamiento de las medidas cuando se tiene la seguridad de que no existe difusión de elementos de un material al otro, se ha preparado una muestra de cobre insertado, mecánicamente, en zinc. La figura 12 muestra la variación de las señales de ${ }^{65} \mathrm{Cu}$ y ${ }^{66} \mathrm{Zn}$ cuando se realizan barridos pasando de un metal al otro. Existe una zona (coincidiendo con el diámetro de cráter de los impactos) en la que las señales de ambos materiales salen mezcladas. Este intervalo está claramente condicionado por el diámetro del impacto láser, por la velocidad de rastreo y por el recorrido entre la cámara láser y la antorcha 


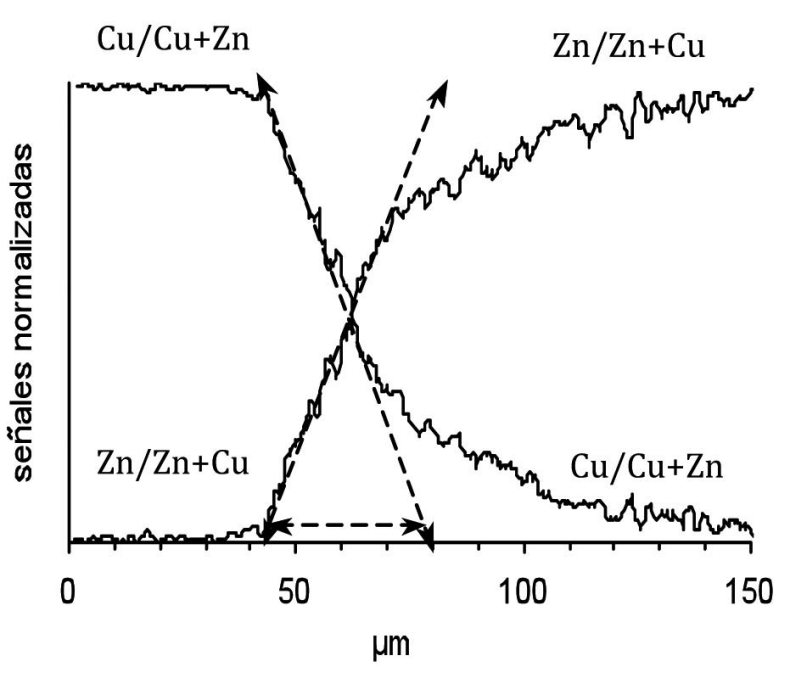

Figura 12. Variación de las señales normalizadas de ${ }^{65} \mathrm{Cu}$ y ${ }^{66} \mathrm{Zn}$ cuando se realizan barridos pasando de un metal al otro.

Figure 12. Normalized signals variation of ${ }^{65} \mathrm{Cu}$ and ${ }^{66} \mathrm{Zn}$ during a continuous line scan $\mathrm{Cu}$ to $\mathrm{Zn}$ and $\mathrm{Zn}$ to $\mathrm{Cu}$.

ICP. Aquí, al no existir la posibilidad de difusión entre ambos metales, este fenómeno puede atribuirse exclusivamente a fenómenos de fragmentación y transporte.

\subsection{Estudios relativos a la resolución en profundidad}

La resolución en profundidad $(\Delta z)$ que puede obtenerse mediante un sistema de ablación por láser, puede determinarse utilizando muestras recubiertas, con capas de recubrimiento de espesores conocidos. Para muestras con un recubrimiento mono-capa, la monitorización de las intensidades de los iones de los elementos en la capa del recubrimiento y la de los elementos del substrato, obtenidos por disparos consecutivos del láser sobre una misma posición, proporcionan el perfil en profundidad de los elementos en ambos materiales, recubrimiento y substrato. En este estudio se han monitorizado, simultáneamente, las señales correspondientes a los elementos níquel del recubrimiento y cobre del substrato.

El objetivo fundamental en el análisis de perfiles en profundidad es el cálculo de la resolución en profundidad, la cual establece el grado de exactitud con que se puede definir una interfase. Las medidas transitorias obtenidas por ICP-MS en "tiempo real" muestran que la disminución de las señales procedentes de los iones presentes en el recubrimiento es más lenta que el aumento de las señales correspondientes a los iones del substrato, las cuales emergen abruptamente cuando el impacto láser alcanza el mencionado substrato. Los cambios en la geometría del cráter y la mezcla del material durante su transporte, desde la cámara de ablación hasta el sistema detector, pueden ser los responsables de las tendencias observadas. En el presente caso, cuando la ablación cesa, la señal medida en el ICP-MS tarda, aproximadamente, $5 \mathrm{~s}$ en bajar hasta el nivel del fondo, lo cual indica que el transporte de muestra es rápido. Por otro lado, un ensayo "blanco" con el gas de transporte, mostró la no existencia de una contaminación apreciable, proveniente del tubo de transporte y de la cámara. De esta observación puede establecerse que la mezcla observada se debe, predominantemente, al propio proceso de ablación. Una correcta optimización de los parámetros operatorios del sistema láser puede minimizar este efecto, mejorando la resolución en profundidad. Así mismo, los parámetros del ICP-MS fueron optimizados para la adquisición de datos, a partir de las medidas de intensidades de los analitos en función del tiempo. Todos los datos han sido recogidos a partir de señales transitorias, en modo altura de pico y con un punto por pico.

En general, los perfiles obtenidos presentan una interfase que aparece ensanchada con respecto a la distribución real de la composición en profundidad. El modo más común de cuantificar el proceso de ensanchamiento es mediante el cálculo de la denominada resolución en profundidad, parámetro que define la exactitud y precisión de la distribución de composición en los perfiles de profundidad obtenidos. La resolución en profundidad, que se puede considerar como la aptitud para distinguir diferencias de la composición a lo largo de una línea perpendicular a una superficie, mide el grado en el cual el experimento es capaz de definir una interfase abrupta. Los conceptos básicos relativos a la definición, significado y evaluación de la resolución en profundidad han sido descritos por Hofmann ${ }^{[26]}$.

\subsubsection{Parámetros que intervienen en el cálculo de la resolución en profundidad}

La resolución en profundidad $(\Delta z)$, se ha evaluado a partir de las señales normalizadas del recubrimiento $\left(\mathrm{N}_{\mathrm{R}}\right)$ y del substrato $\left(\mathrm{N}_{\mathrm{S}}\right)$. El procedimiento seguido para su cálculo, ha sido explicado por Mateo et al. ${ }^{[27]}$. Las señales normalizadas se calculan a partir de las intensidades ICP-MS correspondientes a los 
isótopos medidos en el recubrimiento y en el substrato, en función del número de pulsos $\left(I_{R}\right.$ y $\left.I_{S}\right)$ :

$$
N_{R}=I_{R} /\left(I_{R}+I_{S}\right) \text { y } N_{S}=I_{S} /\left(I_{S}+I_{R}\right)
$$

Para determinar $\Delta z$ es necesario estimar, en primer lugar, la velocidad de ablación (AAR). Dicho parámetro se calcula a partir del espesor del recubrimiento (D) y del número de pulsos requeridos para alcanzar la interfase recubrimiento/substrato $\left(\mathrm{P}_{50}\right): \mathrm{AAR}=$ $\mathrm{D} / \mathrm{P}_{50}$.

La resolución en profundidad viene dada por la ecuación:

$$
\Delta z(\mu \mathrm{m})=\Delta \mathrm{P}(\text { pulsos }) D(\mu \mathrm{m})\left(\mathrm{P}_{50}\right)^{-1} \text { (pulsos) }<>\Delta \mathrm{z}=\Delta \mathrm{PXAAR}
$$

siendo: $\Delta \mathrm{P}$ la diferencia de pulsos láser entre los requeridos para alcanzar el $84 \%\left(\mathrm{P}_{84}\right)$ y el $16 \%\left(\mathrm{P}_{16}\right)$ de dicha señal; $\mathrm{D}$, el espesor del recubrimiento y $\mathrm{P}_{50}$ el número de pulsos requeridos para alcanzar el $50 \%$ de la intensidad normalizada.

La ecuación predice que la mejor resolución en profundidad (p.ej., $\phi z$ menor) se obtiene a partir de las condiciones experimentales para las cuales los valores de $\Delta \mathrm{P}$ y de AAR son más bajos. Los parámetros que intervienen en la determinación de $\Delta z$ aparecen ilustrados en un trabajo previo de Coedo et al. [20]

\subsubsection{Estudio de la influencia de la energía del pulso láser en la velocidad de ablación (AAR) y en la resolución en profundidad $(\Delta \mathrm{z})$}

Los dos parámetros ( $\triangle \mathrm{P}$ y $\mathrm{AAR})$, a partir de los cuales se calcula la resolución en profundidad, vienen condicionados por la densidad de energía o irradiancia, "I" aplicada (energía del pulso láser por unidad de área y por unidad de tiempo), la cual puede calcularse a partir de la siguiente ecuación:

$$
\mathrm{I}\left(\mathrm{GW} \mathrm{cm} \mathrm{cm}^{-2}\right)=\mathrm{E}(\mathrm{mJ}) 410^{5} \Pi^{-1} \mathrm{~d}^{-2}(\mu \mathrm{m}) \omega^{-1}(\mathrm{~ns})
$$

donde, $\mathrm{E}=$ energía del pulso láser; $\mathrm{d}$ = diámetro del cráter y $\omega=$ anchura del pulso láser.

Los parámetros operatorios óptimos, es decir, aquellos que conducen a una mejor resolución en profundidad, se han establecido a partir de los ensayos realizados sobre una muestra compuesta por un substrato de cobre recubierto con una capa de níquel de $25 \mu \mathrm{m}$ de espesor y midiendo los isótopos de mayor abundancia relativa.

Se han evaluado valores de energía del láser de 0,5 a $4 \mathrm{~mJ}$ pulso $^{-1}$ (Irradiancias desde, aproximada-

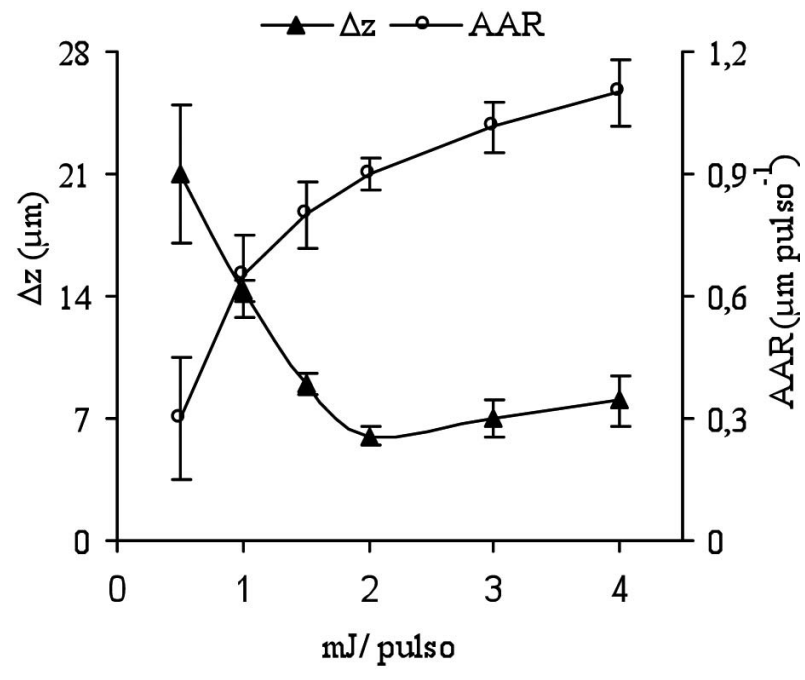

Figura 13. Influencia de la energía láser en AAR and $\Delta \mathrm{z}$.

Figure 13. Influence of laser pulse energy on $A A R$ and $\Delta z$.

mente 1,5 a $9 \mathrm{GW} \mathrm{cm}^{-2}$ ). La figura 13 muestra los valores de AAR y $\Delta z$ en función de la energía del pulso láser. Como muestra la figura, la velocidad de ablación aumenta con la energía, mientras que la resolución en profundidad disminuye, siendo estas variaciones mucho menores a partir de $2 \mathrm{~mJ}$ por pulso (I $\left.\sim 4 \mathrm{GW} \mathrm{cm}^{-2}\right)$. Esta menor influencia puede atribuirse al apantallamiento del plasma que puede tener lugar a densidades de potencia más elevadas y que reduce la cantidad de energía que alcanza la superficie de la muestra. Los elevados valores de las desviaciones estándar para energías de 0,5 mJ por pulso, se deben a la configuración de las señales producidas, que no permiten calcular con precisión los parámetros "P". Debido a que existe una ablación muy débil, y a que su eficiencia no se mantiene al ir profundizando, la señal del cobre empieza a disminuir antes de alcanzar el substrato y la señal del hierro emerge con dificultad, sin llegar a alcanzar un valor estable. Los valores óptimos de resolución en profundidad se han alcanzado con un valor de energía de $2 \mathrm{~mJ}$ pulso.

\subsubsection{Estudio de la influencia de la focalización en la velocidad de ablación (AAR) y en la resolución en profundidad $(\Delta \mathrm{z})$}

El efecto de la focalización se ha estudiado variando la distancia de focalización (DF) entre $\mathrm{DF}=0$, 
focalizando en la superficie (I $\sim 16 \mathrm{GW} \mathrm{cm} \mathrm{cm}^{-2}$ y cráteres con diámetros del orden de $40 \mu \mathrm{m})$ y $\mathrm{DF}=2.000$, focalizando $2.000 \mu \mathrm{m}$ por debajo de la superficie (I $4 \mathrm{GW} \mathrm{cm}{ }^{-2}$ y cráteres con un diámetro del orden de los $120 \mu \mathrm{m})$. La figura 14 muestra dicho efecto en los parámetros AAR y $\Delta z$. Como puede observarse, los valores de AAR disminuyen rápidamente, cuando DF varía de $0 \mathrm{a}+1.000 \mu \mathrm{m}$ y, más lentamente, a partir de $+1.000 \mu \mathrm{m}$, mientras que los valores de $\Delta z$ decrecen lentamente cuando DF varía de $0 \mathrm{a}+750 \mu \mathrm{m}$, para después comenzar a crecer de forma más rápida. Los valores óptimos, mejor resolución en profundidad y menores desviaciones estándar, se han obtenido cuando el haz del láser es focalizado a $750 \mu \mathrm{m}$ (I $8 \mathrm{GW} \mathrm{cm} \mathrm{cm}^{-2}$ y cráteres con un diámetro del orden de los $60 \mu \mathrm{m}$ ), debido a la máxima eficiencia de ablación conseguida en función de la densidad de energía y de la configuración del cráter. A partir de los ensayos realizados, se establecen como parámetros óptimos de la ablación láser los siguientes: Energía del pulso Láser (E): 2 mJ y Focalización (DF): $750 \mu \mathrm{m}$.

\subsubsection{Influencia de la abundancia relativa de los isótopos en los parámetros " $P$ "}

Se ha comprobado que la forma de las señales normalizadas y, en consecuencia, los parámetros "P", están influenciadas por la abundancia relativa de los isótopos naturales utilizados. Las diferentes formas de los perfiles en profundidad, para los dos isótopos del

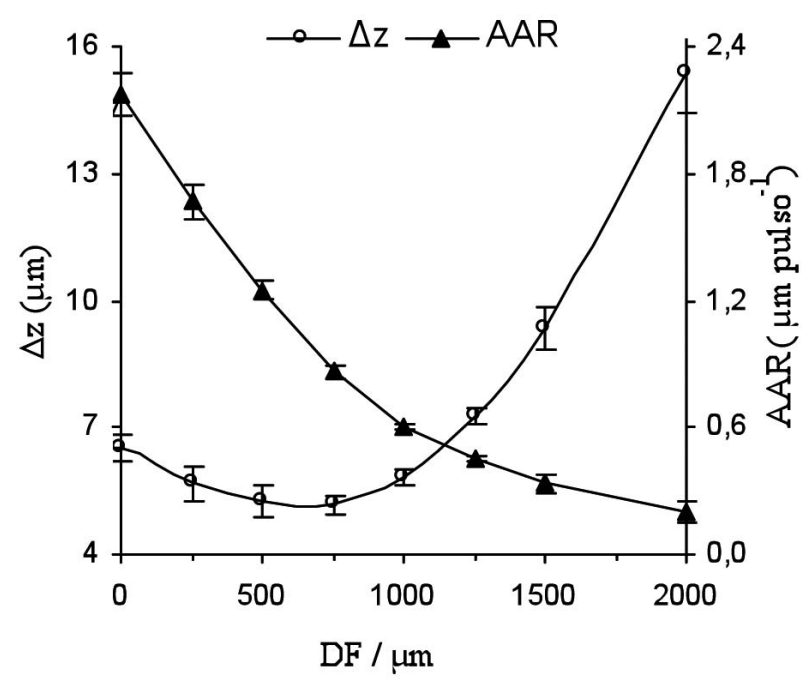

Figura 14. Influencia de la focalización en AAR y $\Delta z$.

Figure 14. Influence of laser focus (DF) on $A A R$ and $\Delta z$. cobre y los dos mayoritarios del níquel, ${ }^{63} \mathrm{Cu}(69 \%)$, ${ }^{65} \mathrm{Cu}(31 \%)$ y ${ }^{58} \mathrm{Ni}(68 \%),{ }^{60} \mathrm{Ni}(26 \%)$, respectivamente, se muestran en la figura 15.

A partir de esta figura se puede calcular el tiempo de perforación $\left(\mathrm{P}_{\mathrm{d}}\right)$, "drill time", definido como número de segundos o de pulsos necesarios para taladrar el recubrimiento y alcanzar el substrato, así como el resto de parámetros "P". Los parámetros $\mathrm{P}_{50}$ y $\Delta \mathrm{P}\left(\mathrm{P}_{84}-\mathrm{P}_{16}\right)$, calculados a partir de la figura 15 , se encuentran representados en la figura 16. Como puede observarse, el parámetro $\mathrm{P}_{\mathrm{d}}$ se mantiene prácticamente constante, no dependiendo de los isótopos considerados, al contrario de lo que ocurre con $\mathrm{P}_{50}$ y $\Delta \mathrm{P}$. Los valores $\mathrm{P}_{50}$ y $\Delta \mathrm{P}$ y, consecuentemente $\Delta \mathrm{P}$, más bajos se corresponden con las señales normalizadas del isótopo con menor abundancia de la capa del recubrimiento y con mayor abundancia del substrato, mientras que los valores más altos corresponden a señales normalizadas para el isótopo con mayor abundancia del recubrimiento y menor abundancia del substrato.

\subsubsection{Estudio sobre muestras con diferentes substratos $y$ recubrimientos}

Las muestras responden a substratos de cobre, aluminio y cromo recubiertos con una capa de níquel (25,

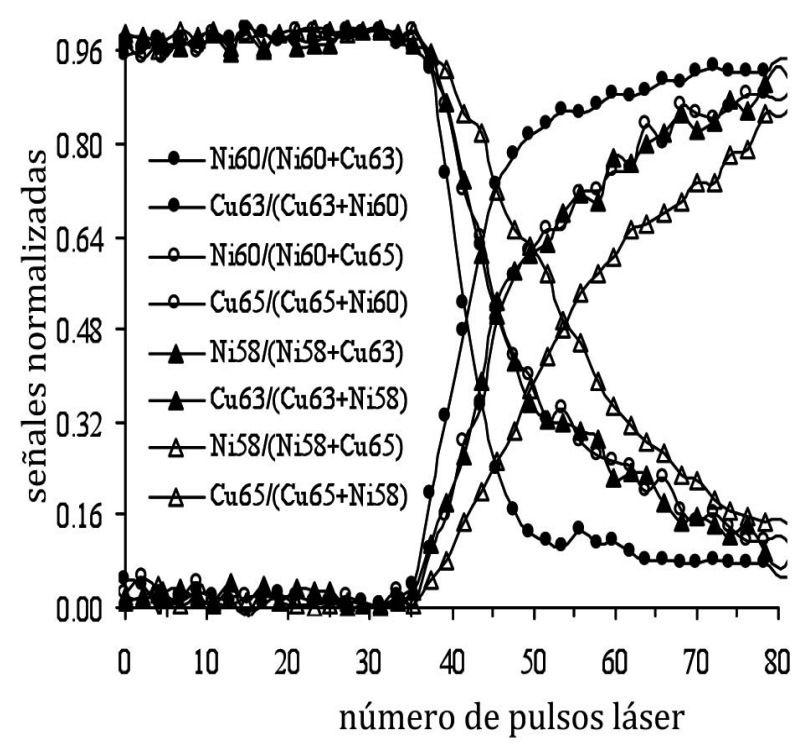

Figura 15. Perfiles en profundidad de las señales normalizadas dependiendo de los isótopos medidos.

Figure 15. Shape of normalized signals profiles depending on the measured isotopes. 


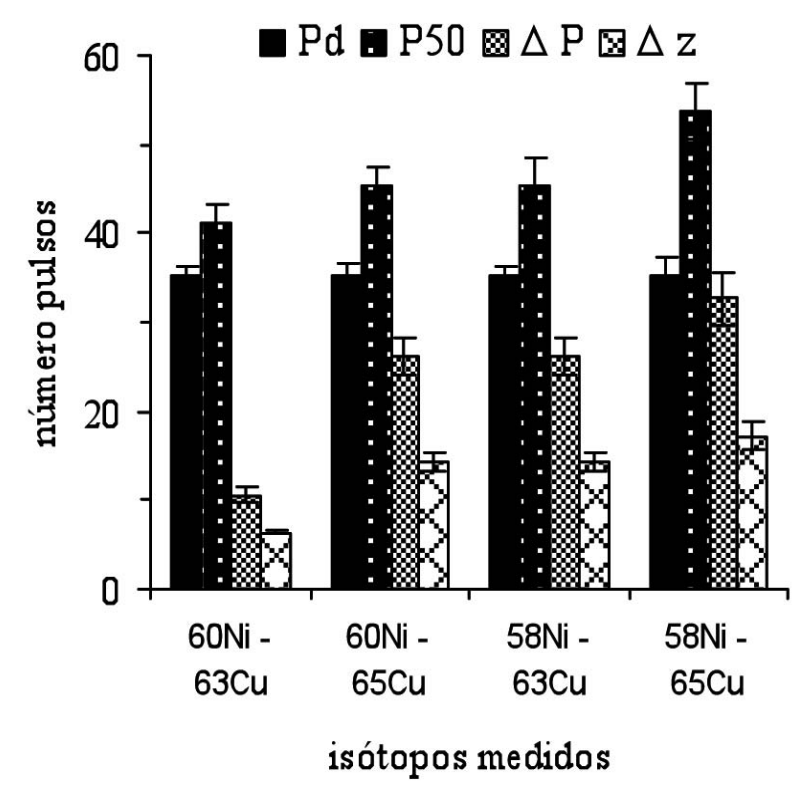

Figura 16. Parámetros "P" dependiendo de la abundancia natural de los isótopos utilizados para obtener las señales normalizadas.

Figure 16. Comparative parameters "P" versus natural abundance of the isotopes analysed.

30 y $20 \mu \mathrm{m}$ de espesor, respectivamente) y substratos de acero con recubrimientos de cobre y zinc (de 22 y $25 \mu \mathrm{m}$, respectivamente).

Todos los isótopos medidos, la relación entre la abundancia relativa del isótopo medido en el recubrimiento (Ar) y el medido en el substrato (As), los valores medios de los parámetros "P" medidos (n $=4$ ), así como los valores calculados de la velocidad de ablación (AAR) y de la resolución en profundidad $(\Delta z)$, se muestran en la tabla IV. Los valores corresponden a una media de 4 medidas. Los valores de $\Delta z$ en función de la relación de la abundancia relativa de los isótopos utilizados en las señales normalizadas se presentan en la figura 17 .

La gráfica muestra que, para todas las muestras, los mejores valores de $\Delta z$ se obtienen para relaciones entre las abundancias relativas de 0,01 a 0,40, independientemente de los isótopos involucrados. Relaciones por encima de 0,4 dan lugar a valores de $\Delta z$ más altos y con desviaciones estándar relativas mayores. En todos los casos, lo más favorable es utilizar el isótopo del substrato con la mayor abundancia relativa e isótopos con abundancia relativa baja, en el recubrimiento. Los resultados muestran, claramente, la influencia de la abundancia relativa de los isótopos medidos, siendo esta influencia más significativa para $\Delta \mathrm{P}\left(\mathrm{P}_{16}-\mathrm{P}_{84}\right)$ que para $\mathrm{P}_{50}$ (del que depende, directamente, $A A R)$.
En la figura 18 se presentan las señales normalizadas obtenidas a partir de los isótopos que originan los valores más bajos de $\Delta z$ con valores de RSD inferiores al 4 \% y las imágenes SEM de los correspondientes cráteres, para las cinco muestras estudiadas.

Los valores de AAR y $\Delta z$ par las cinco muestras estudiadas se presentan comparativamente en la figura 19. Como puede observarse, a excepción de la muestra con substrato de hierro y recubrimiento de zinc, los valores de AAR son todos $<0,70 \mu \mathrm{m}$ por pulso y, consecuentemente, los valores de $\Delta z$ son significativamente menores que los valores de $\triangle \mathrm{P}$. El alto valor de AAR obtenido para el recubrimiento de zinc $(0,95 \mu \mathrm{m}$ por pulso) puede atribuirse a su bajo punto de ebullición $\left(906^{\circ} \mathrm{C}\right)$, en comparación con las temperaturas de ebullición del cobre y níquel $\left(2.595^{\circ} \mathrm{C}\right.$ y $2.730^{\circ} \mathrm{C}$, respectivamente).

\section{CONCLUSIÓN}

Del estudio realizado, se puede establecer que la ablación por láser acoplada a la espectrometría de masas con fuente de plasma de acoplamiento Inductivo, es una técnica apta para la realización de análisis con resolución espacial, tanto lateral como en profundidad.

La resolución lateral está claramente condicionada por el diámetro del impacto láser, por la velocidad de rastreo y por el recorrido entre la cámara láser y la antorcha ICP. Existe una zona, aproximadamente igual al diámetro del cráter, en la que las señales salen mezcladas y no puede establecerse con seguridad que parte se debe a difusión interelemental o a fenómenos de fragmentación y transporte.

En cuanto a la resolución en profundidad, al hacer incidir un láser repetitivamente en una misma posición, sobre la superficie de una muestra, el gráfico de la intensidad de las señales correspondientes a masas atómicas específicas, frente al número de pulsos, proporciona el perfil en profundidad de la muestra. Es necesaria una correcta optimización de los parámetros instrumentales, así como una selección adecuada de los isótopos utilizados para las medidas por ICP-MS.

\section{Agradecimientos}

Este trabajo forma parte de los estudios realizados en el contexto del contrato MAT 2005-00348 subvencionado por la Dirección General de Investigación del Ministerio de Ciencia e Innovación. 
Tabla IV. Isótopos medidos, relación entre la abundancias relativas de los mismos (Ar/As), parámetros "P" $(\mu \mathrm{m}), \mathrm{AAR}\left(\mu \mathrm{m}\right.$ pulso $\left.^{-1}\right)$ y $\Delta \mathrm{z}(\mu \mathrm{m})$.

Table IV. Measured isotopes, relative abundance ratio (Ar/As), "P" parameters ( $\mu m), A A R(\mu m$ pulso $\left.{ }^{-1}\right)$ and $\Delta z(\mu m)$.

\begin{tabular}{|c|c|c|c|c|c|c|c|c|}
\hline Sample & Isotopes & Ar/As & $P_{84}$ & $P_{50}$ & $P_{16}$ & $\Delta \mathbf{P}$ & AAR & $\Delta \mathbf{z}$ \\
\hline \multirow{10}{*}{$\begin{array}{l}\mathrm{Ni} / \mathrm{Cu} \\
(25 \mu \mathrm{m})\end{array}$} & ${ }^{58} \mathrm{Ni} /{ }^{63} \mathrm{Cu}$ & 0,98 & 38,2 & 45,4 & $67^{*}$ & $29^{*}$ & 0,55 & $15,9^{*}$ \\
\hline & ${ }^{60} \mathrm{Ni} /{ }^{63} \mathrm{Cu}$ & 0,38 & 35,7 & 40,3 & 44,0 & 8,3 & 0,62 & 5,14 \\
\hline & ${ }^{62} \mathrm{Ni} /{ }^{63} \mathrm{Cu}$ & 0,053 & 33,8 & 36,2 & 38,5 & 4,7 & 0,69 & 3,24 \\
\hline & ${ }^{61} \mathrm{Ni} /{ }^{63} \mathrm{Cu}$ & 0,017 & $32^{*}$ & 34,7 & 37,0 & $5,0^{*}$ & 0,72 & $3,6^{*}$ \\
\hline & ${ }^{64} \mathrm{Ni} /{ }^{63} \mathrm{Cu}$ & 0,016 & $32^{*}$ & 34,2 & 36,7 & $4,7^{*}$ & 0,73 & $3,4^{*}$ \\
\hline & ${ }^{58} \mathrm{Ni} /{ }^{65} \mathrm{Cu}$ & 2,2 & 40,0 & 50,3 & $74^{*}$ & 34,0 & 0,50 & $17,0^{*}$ \\
\hline & ${ }^{60} \mathrm{Ni} /{ }^{65} \mathrm{Cu}$ & 0,85 & 38,8 & 44,6 & $66^{*}$ & 27,4 & 0,56 & $15,3^{*}$ \\
\hline & ${ }^{62} \mathrm{Ni} /{ }^{65} \mathrm{Cu}$ & 0,12 & 35,0 & 37,3 & 39,2 & 4,2 & 0,67 & 2,81 \\
\hline & ${ }^{61} \mathrm{Ni} /{ }^{65} \mathrm{Cu}$ & 0,038 & $35^{*}$ & 36,7 & 38,5 & $3,5^{*}$ & 0,68 & $2,38^{*}$ \\
\hline & ${ }^{64} \mathrm{Ni} /{ }^{65} \mathrm{Cu}$ & 0,035 & $35^{*}$ & 36,7 & 38,2 & $3,2^{*}$ & 0,68 & $2,17^{*}$ \\
\hline \multirow{5}{*}{$\begin{array}{c}\mathrm{Ni} / \mathrm{Al} \\
(30 \mu \mathrm{m})\end{array}$} & ${ }^{58} \mathrm{Ni} /{ }^{27} \mathrm{Al}$ & 0,68 & 45,5 & 48,4 & $58^{*}$ & $12,5^{*}$ & 0,62 & $7,7^{*}$ \\
\hline & ${ }^{60} \mathrm{Ni} /{ }^{27} \mathrm{Al}$ & 0,26 & 44,8 & 46,9 & 54,4 & 9,6 & 0,64 & 6,14 \\
\hline & ${ }^{62} \mathrm{Ni} /{ }^{27} \mathrm{Al}$ & 0,037 & 42,8 & 44,1 & 47,2 & 4,4 & 0,68 & 2,99 \\
\hline & ${ }^{61} \mathrm{Ni} /{ }^{27} \mathrm{Al}$ & 0,012 & 42,2 & 42,8 & 45,5 & 3,3 & 0,70 & 2,31 \\
\hline & ${ }^{64} \mathrm{Ni} /{ }^{27} \mathrm{Al}$ & 0,011 & 42,2 & 42,8 & 45,2 & 3,0 & 0,70 & 2,10 \\
\hline \multirow{15}{*}{$\begin{array}{c}\mathrm{Ni} / \mathrm{Cr} \\
(20 \mu \mathrm{m})\end{array}$} & ${ }^{58} \mathrm{Ni} /{ }^{52} \mathrm{Cr}$ & 0,81 & 34,2 & $38^{*}$ & $46^{*}$ & $12^{*}$ & 0,52 & $6,24^{*}$ \\
\hline & ${ }^{60} \mathrm{Ni} /{ }^{52} \mathrm{Cr}$ & 0,31 & 33,2 & 37,0 & 40,0 & 6,8 & 0,54 & 3,67 \\
\hline & ${ }^{62} \mathrm{Ni} /{ }^{52} \mathrm{Cr}$ & 0,044 & 30,5 & 31,7 & 35,4 & 4,9 & 0,63 & 3,08 \\
\hline & ${ }^{61} \mathrm{Ni} /{ }^{52} \mathrm{Cr}$ & 0,014 & 30,0 & 30,8 & 33,0 & 3,0 & 0,65 & 1,95 \\
\hline & ${ }^{64} \mathrm{Ni} /{ }^{52} \mathrm{Cr}$ & 0,013 & 30,5 & 31,2 & 33,0 & 2,5 & 0,64 & 1,60 \\
\hline & $58,60 \mathrm{Ni} /{ }^{53} \mathrm{Cr}$ & $>3$ & \multicolumn{6}{|c|}{ no medibles } \\
\hline & ${ }^{62} \mathrm{Ni} /{ }^{53} \mathrm{Cr}$ & 0,38 & 33,0 & 37,0 & 45,0 & 12 & 0,54 & 6,48 \\
\hline & ${ }^{61} \mathrm{Ni} /{ }^{33} \mathrm{Cr}$ & 0,12 & 30,8 & 33,9 & 38,0 & 7,2 & 0,59 & 4,25 \\
\hline & ${ }^{64} \mathrm{Ni} /{ }^{53} \mathrm{Cr}$ & 0,11 & 30,7 & 33,9 & 38,0 & 7,3 & 0,59 & 4,31 \\
\hline & $58,60,62 \mathrm{Ni} /{ }^{50} \mathrm{Cr}$ & $>1$ & \multicolumn{6}{|c|}{ no medibles } \\
\hline & ${ }^{61} \mathrm{Ni} /{ }^{50} \mathrm{Cr}$ & 0,28 & 32,0 & 37,7 & $44^{*}$ & $12,0^{*}$ & 0,53 & $6,36^{*}$ \\
\hline & ${ }^{64} \mathrm{Ni} /{ }^{50} \mathrm{Cr}$ & 0,25 & 32,5 & 37,0 & $43^{*}$ & $10,5^{*}$ & 0,54 & $5,67^{*}$ \\
\hline & ${ }^{58,60,62} \mathrm{Ni} /{ }^{54} \mathrm{Cr}$ & $>1$ & \multicolumn{6}{|c|}{ no medibles } \\
\hline & ${ }^{61} \mathrm{Ni} /{ }^{54} \mathrm{Cr}$ & 0,50 & $30^{*}$ & $38^{*}$ & $46^{*}$ & $16^{*}$ & $0,53^{*}$ & $8,48^{*}$ \\
\hline & ${ }^{64} \mathrm{Ni} /{ }^{54} \mathrm{Cr}$ & 0,45 & $30^{*}$ & $37^{*}$ & $45^{*}$ & $15^{\star}$ & $0,54^{*}$ & $8,10^{*}$ \\
\hline \multirow{3}{*}{$\begin{array}{c}\mathrm{Cu} / \mathrm{Fe} \\
(25 \mu \mathrm{m})\end{array}$} & ${ }^{63} \mathrm{Cu} /{ }^{56} \mathrm{Fe}$ & 0,75 & 41,5 & 43,8 & $57^{*}$ & $15,5^{*}$ & 0,57 & $8,83^{*}$ \\
\hline & ${ }^{65} \mathrm{Cu} /{ }^{56} \mathrm{Fe}$ & 0,34 & 41,2 & 41,7 & 51,2 & 10,0 & 0,60 & 6,00 \\
\hline & ${ }^{63,65} \mathrm{Cu} /{ }^{54,57,58} \mathrm{Fe}$ & $>5$ & \multicolumn{6}{|c|}{ no medibles } \\
\hline \multirow{13}{*}{$\begin{array}{l}\mathrm{Zn} / \mathrm{Fe} \\
(22 \mu \mathrm{m})\end{array}$} & ${ }^{64} \mathrm{Zn} /{ }^{56} \mathrm{Fe}$ & 0,53 & 27,4 & 29,7 & $36^{*}$ & $8,8^{*}$ & 0,74 & $6,5^{*}$ \\
\hline & ${ }^{66} \mathrm{Zn} /{ }^{56} \mathrm{Fe}$ & 0,30 & 26,0 & 28,9 & 34,2 & 8,2 & 0,76 & 6,23 \\
\hline & ${ }^{68} \mathrm{Zn} /{ }^{56} \mathrm{Fe}$ & 0,20 & 23,8 & 25,9 & 29,0 & 5,2 & 0,85 & 4,42 \\
\hline & ${ }^{67} \mathrm{Zn} /{ }^{56} \mathrm{Fe}$ & 0,045 & 22,2 & 23,2 & 25,5 & 3,3 & 0,95 & 3,13 \\
\hline & ${ }^{70} \mathrm{Zn} /{ }^{56} \mathrm{Fe}$ & 0,007 & \multirow{2}{*}{\multicolumn{6}{|c|}{ no medibles }} \\
\hline & $64,66,68 \mathrm{Zn} /{ }^{54} \mathrm{Fe}$ & $>3$ & & & & & & \\
\hline & ${ }^{67} \mathrm{Zn} /{ }^{54} \mathrm{Fe}$ & 0,71 & 23,5 & 30,5 & 35,6 & 12,1 & 0,72 & 8,71 \\
\hline & ${ }^{70} \mathrm{Zn} /{ }^{54} \mathrm{Fe}$ & 0,11 & 22,7 & 24,7 & 27,5 & 4,8 & 0,89 & 4,27 \\
\hline & $64,66,68 \mathrm{Zn} / 57 \mathrm{Fe}$ & $>8$ & \multicolumn{6}{|c|}{ no medibles } \\
\hline & ${ }^{67} \mathrm{Zn} /{ }^{57} \mathrm{Fe}$ & 1,9 & 27,0 & 31,4 & $56^{*}$ & $29^{*}$ & 0,70 & $20,3^{*}$ \\
\hline & ${ }^{70} \mathrm{Zn} /{ }^{57} \mathrm{Fe}$ & 0,28 & 24,2 & 26,5 & 30 & 5,8 & 0,83 & 4,81 \\
\hline & $64,66,68,67 \mathrm{Zn} /{ }^{58} \mathrm{Fe}$ & $>12$ & \multicolumn{6}{|c|}{ no medibles } \\
\hline & ${ }^{70} \mathrm{Zn} /{ }^{58} \mathrm{Fe}$ & 1,9 & 28,0 & 31,4 & $53^{*}$ & $25^{*}$ & 0,70 & $17,5^{\star}$ \\
\hline
\end{tabular}

Valores medios $(n=4)$ con RSD $<7 \%$.

* Parámetros que no se has podido medir con precisión (RSD> 10\%). 


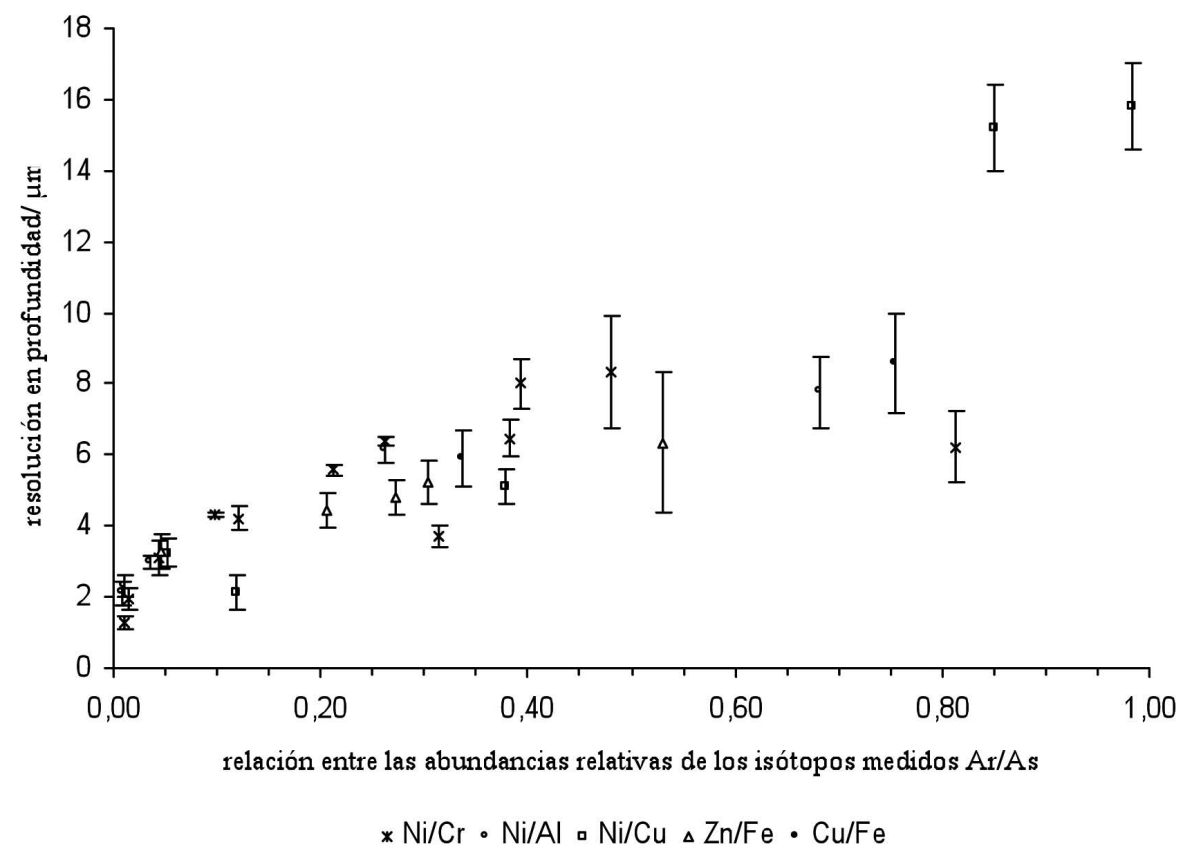

Figura 17. $\Delta z$ en función de la relación entre la abundancia relativa del isótopo medido en el recubrimiento (Ar) y en el substrato (As). Las barras de error corresponden a la desviación estándar para $\mathrm{n}=4$.

Figure 17. $\Delta z$ in function of the relative abundance ratio of the isotope measured in the coating (Ar) and substrate (As). Error bars correspond to $S D$ for $n=4$.
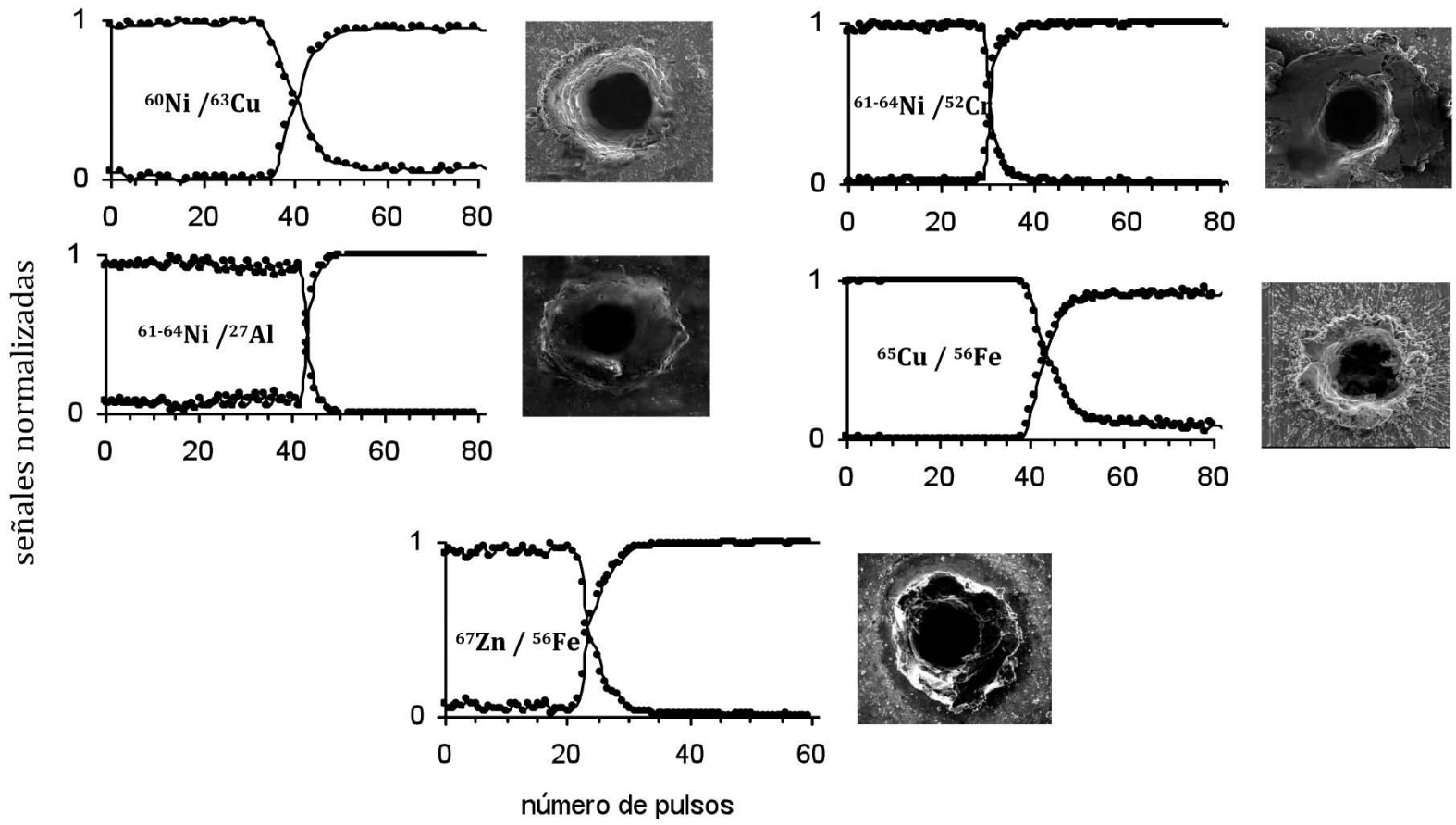

Figura 18. Señales normalizadas de los perfiles en profundidad utilizando los isótopos que dan lugar a los valores más bajos de $\Delta z$ con valores de RSD $<4 \%$ e imágenes SEM de los correspondientes cráteres.

Figure18. Normalized signals in-depth profiles from isotopes providing the lowest $\Delta z$ values with $R S D$ $<4 \%$ and SEM images of craters. 


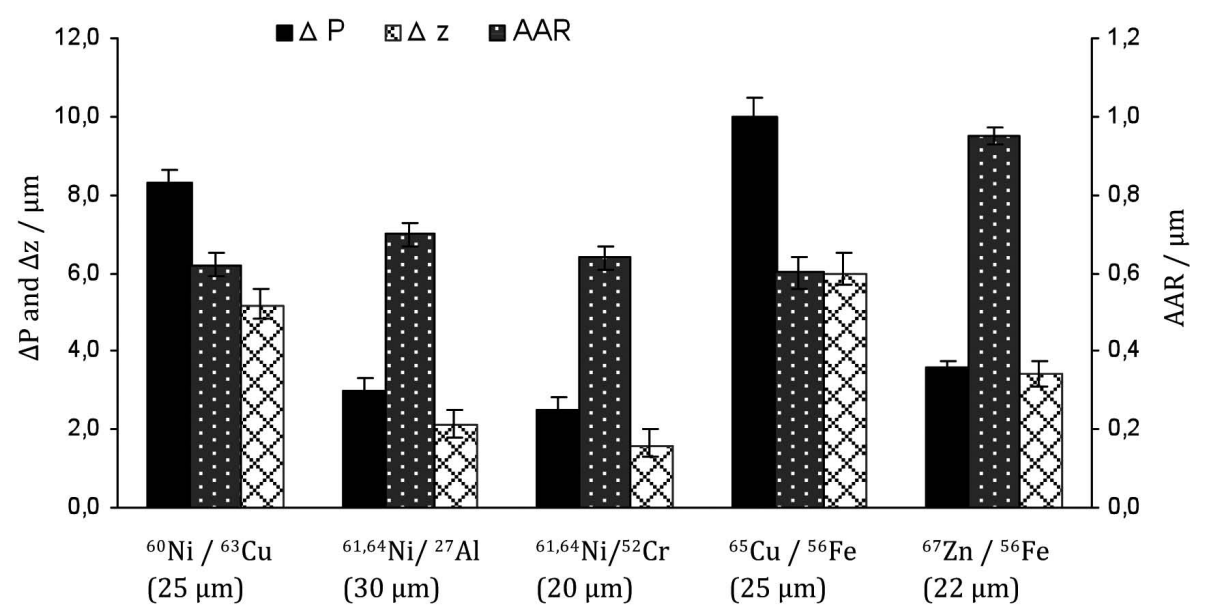

Figura 19. Valores de AAR, $\Delta \mathrm{P}$ y $\Delta \mathrm{z}$ para las cinco muestras estudiadas.

Figure19. Comparative values of $A A R, \Delta P$ and $\Delta z$ for the five studied samples.

\section{REFERENCIAS}

[1] K. Niemax, Fresenius J. Anal. Chem. 370 (2001) 332-340.

[2] D. Günther, S.E. Jackson y H.P. Longerich, Spectrochim. Acta Part B 54 (1999) 381-409.

[3] J.J. González, A. Fernández, X. Maoa y R.E. Russo, Spectrochim. Acta Part B 59 (2004) 369. 374.

[4] I. Kroslakova y D. Günther, J. Anal. Atom. Spectrom. 22 (2007) 51-62.

[5] B. Fernández, F. Claverie, C. Pécheyran, O.F.X. Donard, Trends Anal. Chem. 26 (2007) 951 966.

[6] A. Plotnikov, C. Vogt, K. Wetzig y A. Kyriakopoulos, Spectrochim. Acta Part B 63 (2008) 474 483.

[7] L.I.L. Balcaen, J. Lenaerts, L. Moens y F. Vanhaeke, J. Anal. Atom. Spectrom. 20 (2005) 417-423.

[8] D. Bleiner, P. Lieneman, A. Ulrich, H. Vonmont y A. Wichser, J. Anal. Atom. Spectrom. 18 (2003) 1.146-1.153.

[9] C. Dubuisson, A.G. Cox, C.W. Mcleod, I. Whiteside, R. Jowit y H. Falk, ISIJ Int. 44 (2004) 1.859-1.866.

[10] S. Nafisi, R. Cox y R. Ghomashchi, J. Alloy. Compd. 415 (2006) 99-105.

[11] A.V. Karasev, R. Inoue y H. Suito, ISIJ Int. 41 (2001) 757-765.

[12] A.V. Karasev, y H. Suito, ISIJ Int. 44 (2004) 356-363.

[13] J. Pisonero, I. Kroslakova, D. Günther y C. Latkoczy, Anal. Bioanal. Chem. 386 (2006) 12-20.
[14] C. Latkoczy y T. Ghislain, J. Anal. Atom. Spectrom. 21 (2006) 1.152-1.160.

[15] A.G. Coedo, T. Dorado, I. Padilla y J.C. Fariñas, Talanta 71 (2007) 2.108-2.120.

[16] A.G. Coedo, T. Dorado, I. Padilla y R. Usero, ISIJ Int. 48 (2008) 194-199.

[17] A. Plotnikov, C. Vogt, V. Hoffmann, C. Täschner y K. Wetzig, J. Anal. Atom. Spectrom. 16 (2001) 1.290-1.295.

[18] D. Bleiner, A. Plotnikov, C. Vogt, K. Wetzig y D. Günther, Fresenius J. Anal. Chem., 368 (2000) 221-226.

[19] V.Kanicky, H.R. Kuhn y D. Günther, Anal. Bioanal. Chem. 380 (2004) 218-226.

[20] A.G. Coedo, T. Dorado, I. Padilla y J.C. Fariñas, J. Anal. Atom. Spectrom. 20 (2005) 612-620.

[21] A.V.Izmer, M.V.Zoriy, C. Pickhardt, W. Quadakkers, V. Shemet, L. Singheiser y J.S. Becker, J. Anal. Atom. Spectrom. 20 (2005) 918923.

[22] A. Hrdlicka, V. Otruba, K. Novotny, D. Hünther y V. Kanicky, Spectrochim. Acta, Part B 31 (2005) 307-318.

[23] I. Lieve, L. Balcaen, J. Lenaerts, L. Moensy y F. Vanhaecke, J. Anal. Atom. Spectrom. 20 (2005) 417-423.

[24] M.P.Mateo, C.C. García y R. Hergenröder, Anal. Chem. 79 (2007) 4.908-4.914.

[25] G. Sarah, B. Gratuze y J.N. Barrandon, J. Anal. Atom. Spectrom. 22 (2007) 1.163-1.167.

[26] S. Hofmann, Surf. Interface Anal. 27 (1999) 825-834.

[27] M.P. Mateo, J.M. Vadillo y J.J. Laserna, J. Anal. Atom. Spectrom. 16 (2001) 1.317-1.321. 